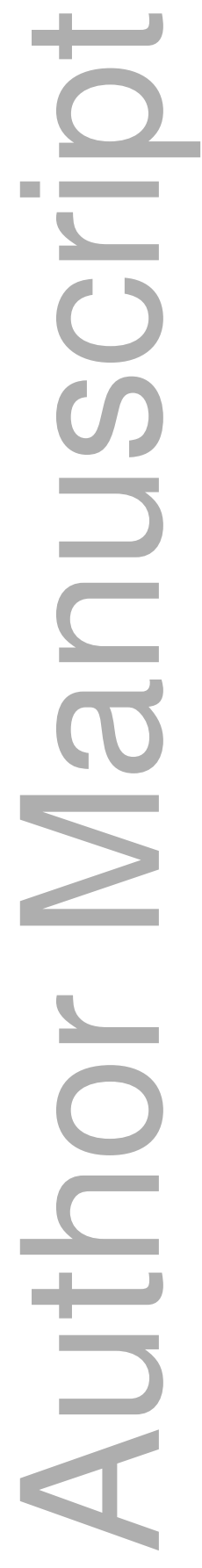

This is the author manuscript accepted for publication and has undergone full peer review but has not been through the copyediting, typesetting, pagination and proofreading process, which may lead to differences between this version and the Version of Record. Please cite this article as doi: $\underline{10.1111 / \text { jmg.12298 }}$

This article is protected by copyright. All rights reserved 


\section{On equilibrium in non-hydrostatic metamorphic systems}

2 R. Powell ${ }^{1}$, K.A. Evans ${ }^{2}$, E.C.R. Green ${ }^{3} \&$ R.W. White ${ }^{4,5}$

$3{ }^{1}$ School of Earth Sciences, University of Melbourne, Vic 3010, Australia

${ }_{4}^{2}$ Dept Applied Geology, Curtin University, Bentley, WA 6845, Australia

${ }_{5}^{3}$ Institute for Geochemistry and Petrology, ETH Zürich, Clausiusstrasse 25, CH 8092,

6 Zürich, Switzerland

7 Institute of Geoscience, University of Mainz, D-55099 Mainz, Germany

${ }^{5}$ now at School of Earth \& Environmental Sciences, University of St Andrews, KY16 9AL,

9 Scotland, UK

Corresponding author: powell@unimelb.edu.au

Short title: Non-hydrostatic metamorphic systems

\section{Abstract}

Metamorphic geology has accumulated a huge body of observation on mineral assemblages that reveal strong patterns in occurrence, summarised for example in the idea of metamorphic facies. On the realisation that such patterns needed a simple explanation, there has been considerable a posteriori success from adopting the idea that equilibrium thermodynamies can be used on mineral assemblages to make sense of the patterns in terms of, for example, the pressure and temperature of formation of mineral assemblages. In doing so, a partieularly simple implicit assumption is made, that mineral assemblages operate essentially hydrostatically. Structural geologists have studied the same rocks for different ends, but, remarkably, the phenomena they are interested in depend on non-hydrostatic stress. We look at the effect of such behaviour on mineral equilibria. With adoption of some plausible assumptions about how metamorphism in the crust works, the consequence of minerals being non-hydrostatically stressed is commonly second order in equilibrium calculations.

KEYWORDS elastic solids; equilibrium thermodynamics; lattice constraint; non-hydrostatic stress 


\section{INTRODUCTION}

In the study of crustal metamorphic rocks, the application of equilibrium thermodynamics hinges on the interpretation of their petrographic features: the mineral assemblages, mineral compositions and mineral textures. This interpretation relates to how the petrographic features form, evolve and are preserved as a rock follows its pressure-temperature path. Since the 1910s, with the classic work of Goldschmidt and Eskola, the striking correspondence and correlation of mineral assemblage with rock-type and orogenic "style" has been used to support the notion that the way to understand metamorphic mineral assemblages is in terms of a preserved equilibrium (e.g. Thompson, 1955; Fyfe, Turner \& Verhoogen, 1958). These observations and ideas form the basis of the metamorphic facies concept (for a historical perspective, see Ch. 1 by F.J. Turner in Fyfe et al., 1958).

The "preserved equilibrium" view of metamorphic mineral assemblages has come to form the status quo in metamorphic geology since that time, underpinning the use of thermobarometry and phase diagrams to determine the "conditions of formation" of rocks (the conditions from where the equilibrium was preserved, primarily the pressure-temperature conditions). However, ideas do surface, or resurface, that challenge the status quo. Such ideas include the effect of non-hydrostatic stress in minerals (e.g. Wheeler, 2014). The importance of such ideas needs to be assessed and the order of magnitude of their likely role evaluated.

As succinctly summarised in the very first section (1.1) of Balluffi, Allen and Carter (2005), on the subject of kinetics and how equilibrium is achieved, most transport phenomena occur more effectively the smaller the length-scale, so equilibrium is more likely to apply on the small scale. Equilibrium at this small scale, if it applies, is called local equilibrium. It was recognised as a guiding principle in material science in the 1940 s (e.g. Darken, 1942), and probably around that time by Korzhinskii in metamorphic geology, as summarised in English in Korzhinskii (1959) calling it mosaic equilibrium (see also Fyfe et al., 1958; Thompson, 1959). The idea of local equilibrium now suffuses all of metamorphic geology, implicitly or explicitly, and underpins the "preserved equilibrium" view of metamorphic mineral assemblages.

The primary justification of adopting a local equilibrium approach to metamorphism is that it provides a simple explanation for the metamorphic patterns observed in metamorphic belts. The main aim of this manuscript is to explore the incorporation of non-hydrostatic thermodynamics into this local equilibrium approach. 


\section{OVERVIEW}

A mineral or rock during orogeny can be considered to behave elasto-viscoplastically (see, for example, de Souza Neto, Perić \& Owen, 2008; Paterson, 2013), with deformation taking place as a consequence of far-field stresses at the elevated temperatures involved. What is observed in a metamorphic rock at the Earth's surface is a complex consequence of its environment and its behaviour during its passage through an orogen. Elasto-viscoplastic processes such as creep are responsible for the development of deformational microstructure. With continuous overprinting, the observed microstructure is what survives after elasto-viscoplastic processes have given way to elastic behaviour as deformation ceases. In the domain of the structural geologist, microstructure can be interpreted in terms of the processes likely to have operated (see for example Paterson, 2013, ch. 5-7). Paterson makes a primary distinction based on the scale of flow sensu lato between processes on atomic, intragranular and granular scales (Paterson, 2013, p. 87). Relevant examples relate to diffusion creep (e.g. Herring, 1950; Coble, 1963), the development of preferred orientation of minerals (e.g. Kamb, 1969; Paterson, 1973), pressure solution (e.g. Rutter \& Elliot, 1976) and grain boundary sliding (e.g. Paterson, 2013, ch. 7). See also Hobbs \& Ord (2015). Viscoplastic processes lead to deformation that is permanent, i.e. it is dissipative (non-conservative), involving entropy production. It is irreversible from a thermodynamic point of view and therefore not a valid subject for the application of equilibrium thermodynamic calculations (e.g. Hobbs \& Ord, 2016).

Dissipative processes are also involved in the chemical equilibration that accompanies elasto-viscoplastic processes, for example the diffusion that flattens chemical potential gradients, nucleation and growth of new minerals, and gain or loss of fluid. Such processes may contribute to microstructure development, and feedbacks between chemical equilibration and deformation are likely to occur at smaller scale with both involving diffusion, e.g. diffusion creep (e.g. Mishin, Warren, Sekerka \& Boettinger, 2013).

With the waning of orogeny, there is a transition from elasto-viscoplastic to elastic behaviour in the minerals and rocks. Elastic behaviour is favoured over elasto-viscoplastic behaviour at lower temperatures, and under fluid-absent conditions. The decrease of far-field stresses when the nature and effect of the boundary conditions of the orogen change, the lowering of temperature with exhumation, and the transition to fluid- or melt-absent conditions, all combine to drive the transition from elasto-viscoplastic to elastic behaviour. Although a non-hydrostatically-stressed elastic solid will tend to relax to a hydrostatically-stressed state given enough time, this time may not be available in relation to the orogenic timescales (e.g. Dabrowski, Powell \& Podladchikov, 2015). In the 
absence of relaxation, non-hydrostatically-stressed minerals may be in equilibrium, and changes to their state can be treated as being thermodynamically reversible, as noted by, for example, McLennan (1980), p106, and discussed by Reiner (1964) and Dealy (2010), and is implicit in material science, for example Li, Oriani and Darken (1966) and subsequent work. After mechanical dissipation has ceased and elastic behaviour predominates, chemical equilibration may continue.

Thus, as a rock passes through an orogeny, it evolves via thermal, mechanical and chemical dissipative processes. Considering a small volume of rock at a point in time, the extant mineral assemblage will depend critically on the rates of the various dissipative processes that contribute towards establishing equilibrium. A possibility is that equilibrium can be established given the time available. If this is the case, and if the mineral assemblage at this point in time could be observed, then equilibrium thermodynamic methods as used in metamorphic petrology could be applied to it. Such methods are applicable only to systems that are not behaving dissipatively, as emphasised by Hobbs and Ord (2016).

The status quo view of metamorphism is that mineral assemblages evolve during orogeny largely at chemical equilibrium on some length scale at least while there is fluid or melt present. This view, an essentially macroscopic view of metamorphism, is commonly implicit, but it underpins application of pseudosections and thermobarometry to mineral assemblages in rocks. Classically, the view relates to what is described as progressive metamorphism, involving essentially-continuous over-printing of mineral assemblages as pressure and temperature change. In a stronger, local-equilibrium form, the over-printing involves continuously-evolving equilibrium mineral assemblages. An argument for this view of metamorphism is that mineral assemblages that appear to satisfy the textural and mineral compositional criteria for equilibrium on some length-scale are preserved as records of the metamorphic process. The majority of what is preserved in mineral assemblages comes from relatively late in a rock's evolution, generally still at elevated temperature judging by the nature of the mineral assemblages preserved (e.g. Guiraud, Powell \& Rebay, 2001; White \& Powell, 2002). How this preservation occurs - how and why mineral assemblages stop eyolving - is a key partly-unanswered question.

To understand the consequences of the idealised situation in which preserved mineral assemblages were in local equilibrium, and in which the minerals were non-hydrostatically-stressed at that time, the equilibrium thermodynamics of non-hydrostatically-stressed elastic solids are considered in the next section. The aim is to establish how important non-hydrostatic stress is for mineral equilibrium calculations. 
Following the presentation of some illustrative calculations, the relevance of the results are discussed briefly.

\section{THERMODYNAMICS OF EQUILIBRIUM}

In this section the equilibrium thermodynamics of elastic crystalline solids (i.e. minerals) that may be hydrostatically or non-hydrostatically stressed is outlined. It is envisaged that the system being considered has reached an equilbrium state, that is, no dissipative processes are operating. The system is made up of grains of one or more solids, and may also include fluid either continuously on grain boundaries, or isolated in pores, or both. Fluid is also referred to as occurring as grains.

\subsection{Types of thermodynamic variable and equilibrium}

There is a distinction between types of thermodynamic variables (e.g. Münster, 1970, $\S 20-21$; Callen, 1985, p35 et seq.). In a fluid the intensive variables, pressure, $p$, temperature, $\theta$, and the chemical potentials, $\mu_{\ell}$, are scalars. Coexisting fluids at equilibrium have the same values of the intensive variables (contact equilibrium of Münster, 1970, p49). At equilibrium, there are no gradients in these intensive variables, and there are no steps in the values of the intensive variables at grain boundaries. There is a conjugate variable to each of these intensive variables, volume, $V$, to $p$, entropy, $S$, to $\theta$, and number of moles of end-member $\ell, n_{\ell}$, to $\mu_{\ell}$. These extensive variables, while being constant in each phase, are proportional to the amount of phase.

In a non-hydrostatically-stressed elastic solid, instead of mechanical equilibrium involving the conjugate pair of scalars $p$ and $V$, the thermodynamics is written in terms of second-rank tensors for stress and strain (see the appropriate sections of a continuum mechanics textbook, for example, Fung \& Tong, 2001; Gurtin, Fried \& Anand, 2010; Lai, Rubin \& Krempl, 2010; Malvern, 1969; Tadmor, Miller \& Elliot, 2012, or Nye, 1985). Minerals do not strain much elastically even with quite large stresses so it is reasonable to use the small strain approximation in the thermodynamics in the way quantified by Gurtin et al., 2010, ch. 52, for example. In the case of small strain, the conjugate pair of variables used are the Cauchy stress tensor, $\mathbf{T}$, and the infinitesimal strain tensor, $\mathbf{E}$ (following the notation of Gurtin et al., 2010, with bold upright case used for second-rank tensors). Whereas mechanical equilibrium in fluid involves constant $p$, the intensive variable, $\mathbf{T}$, need not be constant in an equilibrium (e.g. as illustrated in Llana-Fúnez, Wheeler \& Faulkner, 2012, fig. 1). Instead the criterion for mechanical equilibrium involves the 
divergence of $\mathbf{T}$, with $\operatorname{div} \mathbf{T}=0$, in the absence of body forces, e.g. Gurtin et al., 2010, section 47.2. The probability of spatially heterogeneous stress-strain in an equilibrium complicates the thermodynamics.

The mechanical equilibrium requirement across a grain boundary is a force balance. For two fluids this is simply that their pressures, $p$, are the same. For a solid-fluid boundary, force balance requires that the normal to the boundary must be a principal axis of the stress tensor of the solid, with the magnitude of its principal stress being equal to $p$ in the fluid (e.g. Larché \& Cahn, 1973; Sekerka \& Cahn, 2004; Frolov \& Mishin, 2010).

Solid-solid boundaries across which the lattices are discontinuous are classed as incoherent (e.g. Malvern, 1969), whereas if the lattices of the two grains are continuous with one another, the boundary is coherent. Grain boundaries intermediate between coherent and incoherent are possible, as discussed by Larché and Cahn, 1978, p. 1586. If solid-solid boundaries can slide (referred to as "greased", Leo \& Sekerka, 1989), then the normals to the grain boundary must also be principal axes of the stress tensors in each solid at the point of contact, and these principal stresses identical. If a boundary is deemed not to slide, then the normals to the boundary do not have to correspond to principal axes of the stress tensors in the solids. Constraints on the orientation of principal stresses in solids at grain boundaries can be accommodated because stress can be heterogeneous in solids at equilibrium. The physics becomes more complicated when grain boundaries are curved (e.g. Leo \& Sekerka, 1989; Cermelli \& Gurtin, 1994). Planar incoherent greased grain boundaries are assumed here for illustrative calculation purposes, as in Larché and Cahn, (1973, 1985). Coherent grain boundaries and displacive reactions are not considered.

\subsection{Pressure, thermodynamic pressure, and mean stress}

In the previous section, pressure, $p$, has been used only for a fluid at equilibrium. It has a rigorous definition for fluid that relates to the way internal energy changes with volume, as expanded on below in the Energies of an elastic solid subsection. Because the word, pressure, is used with a less restrictive meaning in the literature, $p$ is referred to here as a thermodynamic pressure, distinct from other usages of the term pressure. The thermodynamic pressure is an intensive variable constant in an equilibrium. A feature of the thermodynamics of solids that can be non-hydrostatically-stressed at equilibrium, is that some of the scalar intensive variables involved are not defined everywhere, including thermodynamic pressure (see later in this subsection), but where scalar intensive variables are defined they are constant in an equilibrium. Solids that can be non-hydrostatically-stressed at equilibrium are referred to simply as solids below. 
In an equilibrium, separate grains of fluid are at the same thermodynamic pressure, $p$. However there is no equivalent scalar intensive variable to thermodynamic pressure within solids. Its role is taken by the stress tensor, $\mathbf{T}$. If, at a point within a solid, the coordinate axes are oriented parallel to the principal axes of $\mathbf{T}$, then the matrix of the stress tensor is diagonal and can be written

$$
\mathbf{T}=\left[\begin{array}{ccc}
t_{1} & 0 & 0 \\
0 & t_{2} & 0 \\
0 & 0 & t_{3}
\end{array}\right]
$$

The mean stress, $\overline{\mathrm{T}}$, is the average of the diagonal elements of the stress tensor, $\mathbf{T}$, $\overline{\mathrm{T}}=\frac{1}{3} \operatorname{tr} \mathbf{T}$, where tr means trace. The mean stress is a third of the first principal invariant of the tensor, which means that it does not depend on the coordinates in which the matrix of the tensor is represented (e.g. Gurtin et al., 2010, section 2.16). If the coordinates are organised as above, $\overline{\mathrm{T}}=\frac{1}{3}\left(t_{1}+t_{2}+t_{3}\right)$. In the literature, negative of mean stress is called various sorts of pressure, or simply pressure, e.g. Connolly (2009), treating compression as negative.

In a solid grain in an equilibrium with fluid, in the limit of non-hydrostatic stress reducing to hydrostatic stress, with the difference between $t_{1}, t_{2}$ and $t_{3}$ getting smaller and smaller, $\overline{\mathrm{T}}$ becomes equal to the pressure of the fluid, $-p$. The stress tensor in the hydrostatically-stressed solid can be written $\mathbf{T}=-p \mathbf{1}$, where $\mathbf{1}$ is the identity tensor, with ones on the diagonal. Alternatively it can be written as $t_{i}=-p$. The mean stress within a hydrostatically-stressed grain is uniform, equal to $-p$.

In contrast to $p$ in a fluid, the mean stress, $\overline{\mathrm{T}}$, in a solid is not a thermodynamic pressure, that is, it is not an intensive variable, constant in an equilibrium. Mean stress varies within a solid in an equilibrium if the solid is heterogeneously-stressed. There will be a step in the mean stress at grain boundaries in all but special cases. For example at the indicated point on the solid-fluid grain boundary in Figure 1, the mean stress in the solid, $\overline{\mathrm{T}}=\left(t_{1}+t_{2}-p\right) / 3$, can be larger or smaller than $p$ in the fluid, depending on the magnitude of $t_{1}$ and $t_{2}$. Here and below, $t_{3}$ is chosen to be the normal to the grain boundary.

Thermodynamic pressure is undefined within solid. A pressure in the solid at grain boundaries, meaning on the surface of the planar interface that is envisaged to represent a grain boundary, is $-t_{3}$ (Larché \& Cahn, 1985, p336-337, corresponding to $-\omega$ in that reference). At solid-fluid boundaries this pressure is equal to $p$, the pressure in the fluid. It is different from the mean stress, the difference being $\left(t_{1}+t_{2}+2 p\right) / 3$. At solid-solid grain boundaries the difference is $\left(t_{1}-t_{3}\right) / 3+\left(t_{2}-t_{3}\right) / 3$ in each solid. In both cases the difference can vary along a grain boundary. Although mean stress is referred to routinely 
as the pressure of a solid, the pressure that appears in the thermodynamics of grain boundaries (in the interface equilibrium relation, see below) is equal to $-t_{3}$.

As in Figure 1, each solid that abuts a fluid grain has a principal stress normal to the grain boundary equal to the negative of the pressure, $-p$, of the fluid grains as discussed above. As $p$ is constant within an equilibrium, these normals have the same magnitude of stress. The non-hydrostatic stress in the solid at a point on a solid-fluid grain boundary can be referred to this $p$ using

$$
q_{1}=t_{1}+p \quad \text { and } \quad q_{2}=t_{2}+p
$$

(Sekerka \& Cahn, 2004). In general $q_{1}$ and $q_{2}$ vary along solid-fluid grain boundaries, with the difference between the mean stress of the solid and the pressure of the fluid being $\frac{1}{3}\left(q_{1}+q_{2}\right)$. If a solid abutting fluid is hydrostatically stressed, then $q_{1}=q_{2}=0$ in the solid at the grain boundary. This $q$ formalism is used below. Some aspects of thermodynamic pressure and mean stress are illustrated in Figure 2.

\subsection{Small strain}

Strain, for example in terms of change of size and shape of the unit cell in a crystalline solid, can be due to factors in addition to externally-applied stress. Such non-elastic strain is referred to as eigenstrain (Mura 1987, p. v), and can arise through for example temperature or composition change. For the small elastic strain in minerals it is reasonable to assume that minerals are linear elastic solids. In the absence of eigenstrain, stress and strain are related linearly by 4 th order tensors, the compliance tensor, $\mathbb{S}$, and the stiffness tensor, $\mathbb{C}$, with the Voigt matrices of the tensors being the inverse of each another (e.g. Nye, 1985, p. 132). The relationships can be written

$$
\mathbf{T}=\mathbb{C E} \quad \text { and } \quad \mathbf{E}=\mathbb{S T}
$$

The components of $\mathbb{S}$ and $\mathbb{C}$ depend on the crystal class of the mineral (e.g. Nye 1985, ch. $8)$.

For the purpose of transparency of development and to see the order of magnitude of effects in illustrative calculations, it is assumed that the solids of interest are isotropic. This means that the number of adjustable parameters is minimised and the algebra is simple. For isotropic solids the principal axes of the strain tensor are coincident with those 
of the stress tensor, so if

$$
\mathbf{T}=\left[\begin{array}{ccc}
t_{1} & 0 & 0 \\
0 & t_{2} & 0 \\
0 & 0 & t_{3}
\end{array}\right] \quad \text { then } \quad \mathbf{E}=\left[\begin{array}{ccc}
e_{1} & 0 & 0 \\
0 & e_{2} & 0 \\
0 & 0 & e_{3}
\end{array}\right]
$$

$\mathbf{T}$ in terms of $\mathbf{E}$, and $\mathbf{E}$ in terms of $\mathbf{T}$, using (2), with the bulk modulus, $\kappa$, and shear modulus, $\mu$, are

$$
t_{i}=(\mathbb{C E})_{i}=\overline{\mathrm{E}}(3 \kappa-2 \mu)+2 \mu e_{i} \quad \text { and } \quad e_{i}=(\mathbb{S T})_{i}=\overline{\mathrm{T}}\left(\frac{1}{3 \kappa}-\frac{1}{2 \mu}\right)+\frac{1}{2 \mu} t_{i}
$$

e.g. Gurtin et al. (2010), sect 52.5, with $\overline{\mathrm{E}}=\frac{1}{3} \operatorname{tr} \mathbf{E}$. For hydrostatic stress, with $t_{1}=t_{2}=t_{3}$, strain is "spherical", $e_{i}=\overline{\mathrm{T}} /(3 \kappa)$, and $\operatorname{tr} \mathbf{E}=\overline{\mathrm{T}} / \kappa$.

For an isotropic solid (and other solids whose crystallographic axes are orthogonal), and with the strain tensor aligned with the crystallographic axes, volume change as a function of strain is

$$
V-V_{0}=V_{0}\left(\left(1+e_{1}\right)\left(1+e_{2}\right)\left(1+e_{3}\right)-1\right) \approx V_{0}\left(e_{1}+e_{2}+e_{3}\right)=V_{0} \operatorname{tr} \mathbf{E}
$$

with $V_{0}$ being the volume at zero strain, and at the temperature and composition of interest (so no eigenstrain is involved). The approximation in (4) results from ignoring terms higher than first order in $e_{k}$, as appropriate for small strain.

For an isotropic solid, referred to a fluid at fixed $p$ at an interface, the volume of the solid is

$$
\frac{V}{V_{0}}=1+\operatorname{tr} \mathbf{E}=1+\frac{1}{\kappa} \overline{\mathrm{T}}=1+\frac{1}{\kappa}\left(-p+\frac{q_{1}+q_{2}}{3}\right)
$$

An alternative derivation of the part of (5) that applies to a hydrostatically-stressed solid, i.e. with $q_{1}=q_{2}=0$, starts with the equation of state for volume in terms of its temperature, $\theta$, and pressure, $p$, at a reference state, $\left\{p_{0}, \theta_{0}\right\}$

$$
d V=V \alpha d \theta+\frac{V}{\kappa} d p
$$

with $\alpha$ the thermal expansion, $1 / V(\partial V / \partial \theta)_{p}$, and $\kappa$ the bulk modulus as above, defined as $-V(\partial p / \partial V)_{\theta}$. The equation of state can be integrated to give $V$ as a function of $p$ and $\theta$ if $\kappa$ and $\alpha$ are constants, and are large and small, respectively

$$
V \approx V_{0}\left(1+\alpha\left(\theta-\theta_{0}\right)-\left(p-p_{0}\right) \frac{1}{\kappa}\right)
$$


with $V_{0}$ the volume at $\left\{p_{0}, \theta_{0}\right\}$, e.g., as in Holland and Powell (1990), p. 91. Commonly used are $p_{0}=0.001 \mathrm{kbar}$ and $\theta_{0}=298 \mathrm{~K}$, with a good approximation being $p_{0} \approx 0$. Then the $p$ term is seen to be the same as in (5), with $\overline{\mathrm{T}}=-p$, while the $\theta$ term is the thermal eigenstrain.

The simplest approximation for the compositional eigenstrain is that the volume of the solid varies linearly with composition between the volumes of its end-members, implying that there is no excess volume of mixing. The thermal and/or compositional eigenstrain need only be considered if the effects of change of temperature and/or composition are required.

\subsection{Lattice constraint}

For mineral equilibria calculations involving non-hydrostatically-stressed minerals, the chemical potentials of the end-members of the minerals need to be formulated. The chemical potentials are derived from an expression for internal energy appropriate for the makeup and behaviour of the solid. The internal energy corresponding to the Gibbs energy used for minerals in petrology, e.g. Powell, White, Green, Holland, and Diener (2014), has a disadvantage in that it involves the implict assumption that the solid is and remains hydrostatically-stressed. Yet solids can be non-hydrostatically-stressed at equilibrium. Even excluding processes involving nucleation such as recrystallisation, equilibration by unrestricted diffusion in the lattice (as in diffusion creep) transforms a solid from being non-hydrostatically stressed to hydrostatically stressed at equilibrium. For elastic solids to hold non-hydrostatic stress at equilbrium, unrestricted diffusion in the lattice needs to be prevented (e.g. Larché \& Cahn, 1973, 1985; Cahn \& Larché, 1983, Mullins \& Sekerka, 1985). Larché and Cahn (1973) consider elastic solids to involve the conservation of lattice points in a reference volume, referred to as the lattice (or network) constraint. Use of the lattice constraint forces substitution of elements on sites in the lattice to be one-for-one: if an element is taken out of a site, another element must be put into it. The lattice constraint precludes unrestricted diffusion, allowing maintenance of non-hydrostatic stress at equilibrium. The lattice constraint of Larché and Cahn (1973) is adopted here for minerals. The lattice constraint is an example of what Gibbs (1906, p58) calls a passive resistance, a feature of a material adopted to prevent a certain process or change. Equilibrium is then subject to that resistance.

Adoption of the lattice constraint has a significant impact on the formulation of equilibrium in elastic solids. With the lattice constraint, in a 1-site solid with atoms of elements substituted on the site, like many alloys as considered by Larché and Cahn 
(1973), the chemical potentials in the solid occur as $\mu$ of element exchanges on the site. These $\mu$ are constant through an equilibrium. The $\mu$ of individual elements do not appear in the thermodynamic description of the solid, only their differences. Extension to minerals and end-members is made in the next paragraph. The $\mu$ in this work are mole-based: the internal energy involved in Larché and Cahn (1973) is per volume, and the derivative of this energy to give chemical potential is with respect to a molar density (moles per volume). The $\mu$ are in $\mathrm{kJ}$ mole ${ }^{-1}$.

The lattice constraint only applies in a solid, given that at grain boundaries, lattice points can be created or lost, for example as a grain grows or is consumed. The distinction between grains and grain boundaries is also fundamental to construction of the equilibrium relations. At grain boundaries, with the lattice constraint not in effect there, the individual $\mu$ are defined and are constant at equilibrium. These individual $\mu$ are consistent with the $\mu$ of exchanges that are constant everywhere within the equilibrium. There is an additional equilibrium relation - the interface equilibrium relation - that connects the thermodynamics of solid with fluid, or solid with solid, across grain boundaries. The interface equilibrium relation is outlined in detail below.

In a 1-site solid, such as an alloy, the chemical potentials are of exchanges of the elements that substitute on the site. The minerals that make up rocks are multi-site, so this needs to be taken into account in applying the lattice constraint to minerals. An end-member of a mineral is a fixed chemical composition 'formula unit' that can be added to or subtracted from the mineral without disrupting its stoichiometry and its charge-balance. Generally this means that end-members have the stoichiometry of the mineral. In a chosen chemical system, the compositional range of a mineral can be completely described using an independent set of end-members in that system (e.g. Powell et al., 2014). For calculations, the system and the range of composition are constrained by the availability of thermodynamic data for the end-members (e.g. Holland \& Powell, 2011; White, Powell, Holland, Johnson \& Green, 2014).

The exchange of formula units of end-members in minerals is the lattice constraint equivalent of exchanging elements on a site, as in Larché and Cahn (1973). End-members can be chosen to represent such an exchange. Taking plagioclase as an example, for all standard calculation purposes potassium-free plagioclase can be treated as a binary, in terms of the additive end-members $\mathrm{NaAlSi}_{3} \mathrm{O}_{8}$ (albite, ab) and $\mathrm{CaAl}_{2} \mathrm{Si}_{2} \mathrm{O}_{8}$ (anorthite, an), in $\mathrm{CaO}-\mathrm{Na}_{2} \mathrm{O}-\mathrm{Al}_{2} \mathrm{O}_{3}-\mathrm{SiO}_{2}$. Now, from a lattice constraint point of view, there is one thermodynamically relevant end-member, the exchange end-member, $(\mathrm{CaAl})_{-1}(\mathrm{NaSi})$. Internal equilibrium in a plagioclase grain is defined by constant $\mu_{(\mathrm{CaAl})_{-1}(\mathrm{NaSi})}$. The 
chemical potentials of the additive end-members, $\mu_{\mathrm{CaAl}_{2} \mathrm{Si}_{2} \mathrm{O}_{8}}$ and $\mu_{\mathrm{NaAlSi}_{3} \mathrm{O}_{8}}$, are not defined in a plagioclase grain. However, at a grain boundary, both $\mu_{\mathrm{CaAl}_{2} \mathrm{Si}_{2} \mathrm{O}_{8}}$ and $\mu_{\mathrm{NaAlSi}_{3} \mathrm{O}_{8}}$ are defined, and are consistent with $\mu_{(\mathrm{CaAl})_{-1}(\mathrm{NaSi})}=\mu_{\mathrm{NaAlSi}_{3} \mathrm{O}_{8}}-\mu_{\mathrm{CaAl}_{2} \mathrm{Si}_{2} \mathrm{O}_{8}}$ from the internal equilibrium in the grain.

In a solid with $n$ independent additive end-members, one of them, say $\ell$, can be used as the common end-member in the exchanges. Then there is a set of independent $n-1$ exchange end-members, $\ell_{-1} k$. Internal equilibrium is defined by constant $\mu_{\ell_{-1} k}$. At a solid-fluid grain boundary, as well as $\mu_{\ell_{-1} k}$ being defined via the internal equilibrium, the interface equilibrium relation allows $\mu_{\ell}$ to be determined, so that the chemical potentials of all of the additive end-members can be generated. Any end-member can be $\ell$.

Now, a simpler notation is adopted for the end-member exchange variable, with $\ell_{-1} k$ defined as $k \ell$, and $\mu_{\ell_{-1} k}$ defined as $\mu_{k \ell}$. So, for example, in plagioclase, $\mu_{(\mathrm{CaAl})_{-1}(\mathrm{NaSi})}$ is $\mu_{\mathrm{ab} a n}$, noting that the use of the plagioclase end-member name in the $\mu$ subscript means the composition of the formula unit with no implication of mineral structure. So $\mu_{\mathrm{aban}}^{\mathrm{f}}$, with superscript $f$ meaning fluid, means $\mu_{(\mathrm{CaAl})_{-1}(\mathrm{NaSi})}^{\mathrm{f}}$. Also $\mu_{\mathrm{ab} \text { an }}=\mu_{\text {pa ma }}$ by definition, given that both exchanges reduce to the same exchange, $(\mathrm{CaAl})_{-1}(\mathrm{NaSi})$, with pa $=$ paragonite and $m a=$ margarite

\subsection{Energies of an elastic solid}

In this subsection, and the remainder of the section, a solid is assumed to be a linear hyperelastic elastic crystalline solid in which the lattice constraint applies, with the term hyperelastic defined below. Depending on context, this sort of material is referred to as "solid" or "elastic solid".

In equilibrium thermodynamics, a fundamental equation relates the internal energy, $U$, to a sufficient set of extensive variables. Whereas for a fluid, this fundamental equation involves only scalars (e.g. Alberty, 2001, eq. 1.1-2), for an elastic solid it does not, as discussed above. Instead, the fundamental equation involves the strain tensor, $\mathbf{E}$ and the stress tensor, T (Gurtin et al., 2010, section 52.3), for small strain. For an arbitrary amount of homogeneously-stressed lattice-constraint solid, a sufficient set of extensive variables to consider the variation of the internal energy is entropy, $S$, strain, $\mathbf{E}$, and the number of moles of exchange end-members. $V_{0}$ is the volume of solid in the reference state: zero stress, at specified $\theta$ and composition. Then,

$$
d U=\left(\frac{\partial U}{\partial S}\right)_{\mathbf{E}, n_{i}} d S+\left(\frac{\partial U}{\partial \mathbf{E}}\right)_{S, n_{i}}: d \mathbf{E}+\sum_{k \neq \ell}\left(\frac{\partial U}{\partial n_{k \ell}}\right)_{\mathbf{E}, S, n_{i \ell(i \neq k)}} d n_{k \ell}
$$


with $n_{k \ell}$ the number of moles of exchange end-member, $k$. The sum is over an independent set of exchanges. For a 1-end-member solid, there is no sum term as there are no exchanges. The colon symbol denotes the tensor inner product, with $\mathbf{A}: \mathbf{B}=\sum \sum A_{i j} B_{i j}$ or in Einstein notation, $A_{i j} B_{i j}$. The variations in (7), like $d S$, relate strictly to quasi-static processes, e.g. Callen (1985), Sect. 4.2. The derivatives in (7) can be identified with intensive variables, with the terms involving conjugate pairs of intensive and extensive variables, e.g Callen (1985), Sect. 12-3, giving

$$
d U=\theta d S+V_{0} \mathbf{T}: d \mathbf{E}+\sum_{k \neq \ell} \mu_{k \ell} d n_{k \ell}
$$

in which the $\mu_{k \ell}$ are chemical potentials of exchange end-members, $k \ell$, involving end-member $k$ substituting for end-member $\ell$, as introduced in the Lattice constraint section above. Note that

$$
\mathbf{T}=\frac{1}{V_{0}}\left(\frac{\partial U}{\partial \mathbf{E}}\right)_{S, n_{k \ell}}
$$

e.g. Gurtin et al. (2010), eq. 52.35. Relations such as $(8,9)$ define hyperelasticity, the subset of elastic behaviours for which $(8,9)$ are true (e.g. Gurtin et al., 2010, p279).

In (8), $V_{0} \mathbf{T}$ : $d \mathbf{E}$ replaces $p d V$ in the expression for $d U$ for a fluid, where the thermodynamic pressure in the fluid is defined by $p=-(\partial U / \partial V)_{S, n_{i}}$, (e.g. Callen, 1985, eq. 2.2). There is no equivalent derivation of a thermodynamic pressure within an elastic solid. Whereas $(\partial U / \partial V)_{S, \mathbf{E}_{0}, n_{i}}$, with the differentiation at constant deviatoric strain, $\mathbf{E}_{0}=\mathbf{E}-\frac{1}{3} \operatorname{tr} \mathbf{E}$, gives the mean stress (e.g. McLellan, 1980, eq. 8.3.2), this is not a thermodynamic pressure by the definition in the Pressure section above (c.f. Hobbs \& Ord, 2016, p. 200). Indeed, pressures with a range of different definitions can be generated, depending on what is held constant in $\partial U / \partial V$ instead of $\mathbf{E}_{0}$. None of these are spatially constant within an equilibrium so none are thermodynamic pressures.

Legendre transforms allow energies to be generated that make the thermodynamics more easily used. The Legendre transform allows the variables in a conjugate pair in $d U$ to be interchanged (Cällen, 1985, p. 141 et seq.; Alberty, 2001). A Legendre transform of $U$ with respect to $\theta$, denoted $\mathcal{L}_{\theta}$, gives a new energy, the Helmholtz energy, $F=\mathcal{L}_{\theta} U$ (e.g. McLellan, 1980, eq. 10.2.7)

$$
d F=-S d \theta+V_{0} \mathbf{T}: d \mathbf{E}+\sum \mu_{k \ell} d n_{k \ell}
$$

In a system at specified $\theta$ (rather than $S$ ), the Helmholtz energy (rather than $U$ ) is minimised at equilibrium. Gibbs energy is defined by a Legendre transform relating to the 
mechanical term in $U$. For a solid, Gibbs energy, $G=\mathcal{L}_{\mathbf{T} \theta} U$ (e.g. McLellan, 1980, eq. 10.2.11), is

$$
d G=-S d \theta-V_{0} \mathbf{E}: d \mathbf{T}+\sum \mu_{k \ell} d n_{k \ell}
$$

A Maxwell relation generated from this equation is used below. For a fluid, $G$ is defined as $\mathcal{L}_{p \theta} U$. However $\mathcal{L}_{p \theta} U$ cannot be used as a definition for $G$ for an elastic solid because a thermodynamic pressure, $p$, is not defined in such solids.

The Gibbs-Duhem relation is an important relationship that indicates that at equilibrium the intensive variables cannot be varied independently. It arises from a complete Legendre transform of $d U$. For an homogeneously-stressed part within a solid the Gibbs-Duhem relation is

$$
0=-S d \theta-V_{0} \mathbf{E}: d \mathbf{T}+\sum n_{k \ell} d \mu_{k \ell}
$$

This also applies within a hydrostatically-stressed solid (Johnson \& Schmalzried, 1992). Temperature, mean stress and the chemical potentials can be independently varied in a hydrostatically-stressed solid as $\mathbf{T}$ is only partly characterised by $\overline{\mathbf{T}}$. This result is in contrast to fluids in which temperature, thermodynamic pressure and the chemical potentials cannot be varied independently.

\subsection{Chemical equilibrium involving an elastic solid}

The chemical potentials that are defined play a central role in characterising equilibrium because they are constant in an equilibrium. The relationships between chemical potential and mineral composition allow the compositions of minerals at equilibrium to be calculated. In a solid, chemical potentials of exchange end-members are defined and are constant. These can be used to look at the relationship between variations in stress and mineral composition. In a $n$-end-member solid there are $n-1$ independent exchanges, and $n-1$ independent composition parameters. Different solid grains (i.e. different minerals) that are in equilibrium and involve the same exchanges will have the same $\mu$ of the exchanges.

In order to combine information from all of the end-members, not just exchange ones, the equilibrium in fluid and in solid-solid grain boundaries have to be determined. In these locations the chemical potentials of all the end-members are defined. The interface equilibrium relation - outlined later in this subsection - allows the extraction of the information on the chemical potentials of the individual additive end-members from the chemical potentials of the exchange end-members. Moreover it allows the chemical 
potential of the end-member in a 1-end-member solid, for example, $\mathrm{SiO}_{2}$ in quartz, to be determined in fluid or at grain boundaries, reflecting the presence of, and equilibrium with, quartz, even though $\mu_{\mathrm{SiO}_{2}}$ is not defined in quartz.

In a binary elastic solid, there is one chemical potential of an exchange end-member, $\mu_{12}$, in (11), with $n_{12}$ the number of moles of the exchange end-member

$$
\mu_{12}(\mathbf{T})=\left(\frac{\partial G}{\partial n_{12}}\right)_{\mathbf{T}, \theta}
$$

written as $\mu_{12}(\mathbf{T})$ to indicate that this is a function of the state of stress, $\mathbf{T}$. To make calculations, it is advantageous to write $\mu_{12}(\mathbf{T})$ in terms of the properties of the hydrostatically-stressed additive end-members, 1 and 2, as might be gleaned from a thermodynamic dataset. The approach taken follows that of Larché and Cahn (1985), Sect. 4.2 , with equation numbers in square brackets here indicating the equations in that reference. A Maxwell relation, [4.13], derived from the differential form of $G$ shown in equation (11), [4.12], with $G$ called $\phi$ in Larché and Cahn (1985)

$$
V_{\ominus}\left(\frac{\partial \mathbf{E}}{\partial n_{12}}\right)_{\mathbf{T}, \theta}=-\left(\frac{\partial \mu_{12}(\mathbf{T})}{\partial \mathbf{T}}\right)_{\theta, n_{12}}
$$

with $V_{\ominus}$ being the volume in the $\ominus$ reference state, see below. This equation says that the dependence of strain on composition is related to the dependence of chemical potential on stress. Integrating (14) from a reference state to the conditions of interest gives the corresponding strain as a function of composition. The most convenient reference state is the unstressed solid $(\mathbf{T}=\mathbf{0})$, at the temperature of interest, and for a reference composition, taken to be that of pure end-member 2. This reference state is denoted by the subscript, $\ominus$. The conventional reference state involving $\theta_{0}=298 \mathrm{~K}$ is denoted by subscript 0 , with the transition between the two reference states, $\ominus$ and 0 , being via the thermal eigenstrain.

The strain, E, can be decomposed into two parts (Larché \& Cahn, 1973, p1056). The first is the compositional eigenstrain, $\mathbf{E}_{\mathbf{c}}$, associated with change of composition from that of $V_{\ominus}$ to the composition of interest. The second part is the strain from the stress, $\mathbf{E}-\mathbf{E}_{\mathrm{c}}$. For linear elastic solids, $\mathbf{E}=\mathbf{E}_{\mathbf{c}}+\mathbb{S} \mathbf{T},[4.15]$.

Integrating the Maxwell relation, (14), from the unstressed state $(\mathbf{T}=\mathbf{0})$ to the state of stress of interest, $\mathbf{T}$, given strain as a function of composition, to give $\mu$ as a function of stress at the composition of interest is

$$
\int_{\left.\mu_{12}\right|_{\mathbf{0}}}^{\left.\mu_{12}\right|_{\mathbf{T}}} d \mu_{12}(\mathbf{T})=-V_{\ominus} \int_{\mathbf{0}}^{\mathbf{T}}\left(\frac{\partial \mathbf{E}}{\partial n_{12}}\right): d \mathbf{T}
$$


the derivative being at constant $\theta$. Substituting $\mathbf{E}=\mathbf{E}_{\mathbf{c}}+\mathbb{S} \mathbf{T}$, and integrating

$$
\mu_{12}(\mathbf{T})-\left(\mu_{12}\right)_{\ominus}=-V_{\ominus}\left(\frac{\partial \mathbf{E}_{\mathrm{c}}}{\partial n_{12}}\right): \mathbf{T}-V_{\ominus} \frac{1}{2}\left(\frac{\partial \mathbb{S}}{\partial n_{12}}\right) \mathbf{T}: \mathbf{T}
$$

in which $\left(\mu_{12}\right)_{\ominus}$ is the chemical potential in the reference state. The derivative of $\mathbb{S}$ is the 4 th rank tensor whose elements are the composition-dependence of the elements of $\mathbb{S}$. For an isotropic solid, the composition-dependence is of the bulk and shear moduli that make up $\mathbb{S}$.

For the illustrative calculations below, approximations can be made to simplify this equation, Appendix 1, (34). The aim is to be able to write chemical potentials of exchanges in non-hydrostatically-stressed solid in terms of properties of hydrostatically-stressed additive end-members. Then, for a standard state of the hydrostatically-stressed pure end-member at the temperature of interest and an arbitrary pressure, $P,[4.23]$

$$
\mu_{12}(\mathbf{T})=G_{1}(P)-G_{2}(P)+\mathrm{R} \theta \ln \frac{a_{1}}{a_{2}}-V_{12}(P+\overline{\mathrm{T}})
$$

with $G_{1}(P)$ and $G_{2}(P)$ the Gibbs energies of end-members 1 and 2 at $P$ and $\theta, a_{1}$ and $a_{2}$ the activities of 1 and 2 , and $V_{12}$ the difference between the volumes of 1 and $2, V_{1}-V_{2}$. This equation, (16), gives the chemical potential of the exchange end-member, 12 , in the non-hydrostatically-stressed solid (as specified by $\mathbf{T}$ ) in terms of the properties of the end-members 1 and 2 , in the $G$ and $V_{12}$, and the composition of the solid in the ratio of the activities, $a_{1} / a_{2}$. The pressure in (16) is chosen for the application of interest. At a solid-fluid grain boundary, $P$ can be the pressure in the fluid, so $P=p$. For calculations within a solid, $P=-\overline{\mathrm{T}}$ or $P=0$ are obvious choices.

\subsection{Chemical equilibrium at grain boundaries}

At grain boundaries the lattice constraint is not active, and there the lattice can be created or consumed. So, along grain boundaries and within fluid the chemical potentials of the individual additive end-members are defined, and are constant at equilibrium (Larché \& Cahn, 1973). An exchange $\mu$ in a grain boundary has the same constant value that it has in the solids and fluid at equilibrium, as illustrated in Figure 3. In this Figure, the end-member $\mu$ are arranged so that the differences between $\mu_{\mathrm{ab}}$ and $\mu_{\mathrm{an}}$, and between $\mu_{\mathrm{pa}}$ and $\mu_{\mathrm{ma}}$ are the same, and the same as the exchange potential of $\mu_{(\mathrm{CaAl})_{-1}(\mathrm{NaSi})}$, which is the same as $\mu_{\mathrm{ab} \text { an }}$ and $\mu_{\mathrm{pama}}$.

Doing conventional calculations on a mineral assemblage involves (or can be reduced to) writing the chemical potentials of all of the end-members of the minerals and fluid in an 
equilibrium, or at least those end-members for which there are data in a thermodynamic dataset. If all phases are hydrostatically-stressed then the chemical potentials apply everywhere in the phases, while also applying at grain boundaries. However if any solids are non-hydrostatically-stressed, the chemical potentials of their additive end-members are only defined at grain boundaries or in the fluid. So, for example, the effect of having a non-hydrostatically-stressed grain of the 1-end-member solid, quartz, in a mineral assemblage is only seen in the grain boundaries or fluid, meaning that the presence of quartz allows a $\mu_{\mathrm{SiO}_{2}}$ to be defined in the grain boundaries and in fluid, even though $\mu_{\mathrm{SiO}_{2}}$ in the quartz grain is not defined. Considering plagioclase, at equilibrium $\mu_{(\mathrm{CaAl})_{-1}(\mathrm{NaSi})}$ is defined and constant everywhere, but $\mu_{\mathrm{CaAl}_{2} \mathrm{Si}_{2} \mathrm{O}_{8}}$ and $\mu_{\mathrm{NaAlSi}_{3} \mathrm{O}_{8}}$ separately are only defined at grain boundaries (interfaces) or in fluid. It is the interface equilibrium relation that allows these separate $\mu$ to be calculated, stemming originally from Gibbs (1906), eq. 388 .

The interface equilibrium relation for a binary elastic solid in equilibrium with a fluid is given by Larché and Cahn (1985), [3.14], incorporating [3.18]. Per mole

$$
F(\mathbf{T})+p V(\mathbf{T})-c \mu_{1}^{f}-(1-c) \mu_{2}^{f}=0
$$

with $F(\mathbf{T})$ and $V(\mathbf{T})$ being for the solid, $p$ the pressure of the fluid at the interface, $\mu_{1}^{f}$ and $\mu_{2}^{f}$ the chemical potentials of 1 and 2 in the fluid, and $c$ is the proportion of end-member 1 in the solid. A more useful form of the interface equilibrium relation can be made using the fact that the chemical potential of exchange of 1 with 2 in the solid, $\mu_{12}^{s}(\mathbf{T})$, is equal to $\mu_{1}^{f}-\mu_{2}^{f}$ in the fluid at the solid-fluid interface

$$
F(\mathbf{T})+p V(\mathbf{T})-c \mu_{12}^{s}(\mathbf{T})=\mu_{2}^{f}
$$

adding a superscript $s$ to the $\mu$ for solid. Using the result for $F(\mathbf{T})+p V(\mathbf{T})$ from Appendix $2,(41)$, and the result for $\mu_{12}(\mathbf{T})$ in (16), with $G(p)$ being at $p$ and $\theta,(17)$ becomes

$$
\begin{aligned}
\mu_{2}^{f}= & c\left(G_{1}^{s}(p)+\mathrm{R} \theta \ln a_{1}^{s}\right)+(1-c)\left(G_{2}^{s}(p)+\mathrm{R} \theta \ln a_{2}^{s}\right)+ \\
& \frac{V_{0}}{18 \kappa}\left(q_{1}+q_{2}\right)^{2}+\frac{V_{0}}{6 \mu}\left(q_{1}^{2}-q_{1} q_{2}+q_{2}^{2}\right)- \\
& c\left(G_{1}^{s}(p)+\mathrm{R} \theta \ln a_{1}^{s}-\left(G_{2}^{s}(p)+\mathrm{R} \theta \ln a_{2}^{s}\right)-V_{12}(p+\overline{\mathrm{T}})\right)
\end{aligned}
$$

with $V_{0}$ the volume of the solid, unstressed at $\theta$ and at the composition of interest. Simplifying (18), using $p+\overline{\mathrm{T}}=\frac{1}{3}\left(q_{1}+q_{2}\right)$

$$
\mu_{2}^{f}=G_{2}^{s}(p)+\mathrm{R} \theta \ln a_{2}^{s}+\frac{1}{3} c\left(q_{1}+q_{2}\right) V_{12}+\frac{V_{0}}{18 \kappa}\left(q_{1}+q_{2}\right)^{2}+\frac{V_{0}}{6 \mu}\left(q_{1}^{2}-q_{1} q_{2}+q_{2}^{2}\right)
$$


In combination with $\mu_{12}^{f}=\mu_{12}^{s}$ from (16), and $\mu_{2}^{f}$ from (19), the chemical potential of the other end-member in the binary is

$$
\mu_{1}^{f}=G_{1}^{s}(p)+\mathrm{R} \theta \ln a_{1}^{s}-\frac{1}{3}(1-c)\left(q_{1}+q_{2}\right) V_{12}+\frac{V_{0}}{18 \kappa}\left(q_{1}+q_{2}\right)^{2}+\frac{V_{0}}{6 \mu}\left(q_{1}^{2}-q_{1} q_{2}+q_{2}^{2}\right)
$$

Although only the chemical potential of the exchange is defined in the solid, the chemical potentials of the two additive end-members are defined in the fluid by (19-20). The chemical potentials are constant in the fluid and in grain boundaries at equilibrium.

For a 1-end-member solid involving end-member 1 the equivalent of (17) is $F(\mathbf{T})+p V(\mathbf{T})=\mu_{1}^{f}$, which becomes, with the result from Appendix 2

$$
\mu_{1}^{f}=G_{1}^{s}(p)+\frac{V_{0}}{18 \kappa}\left(q_{1}+q_{2}\right)^{2}+\frac{V_{0}}{6 \mu}\left(q_{1}^{2}-q_{1} q_{2}+q_{2}^{2}\right)
$$

with $V_{0}$ the volume of end-member 1 , unstressed at $\theta$. Although the chemical potential of the end-member is not defined in the solid, the chemical potential of the end-member is defined in the fluid by (21). The chemical potential is constant in the fluid and in grain boundaries at equilibrium.

The expressions, (19-21), apply at grain boundaries, so the chemical potentials are written for the compositions of the solids there, as well as the stress relationships represented by the terms in $q_{1}$ and $q_{2}$. As $q_{1}$ and $q_{2}$ in a solid may vary along a grain boundary, constant $\mu_{\ell}^{f}$ along the boundary means that $q_{1}$ and $q_{2}$ are constrained to covary.

The logic of (19-21) can be adapted to consider solid-solid grain boundaries (Larché \& Cahn, 1985, sect. 3.5.2). Along solid-solid grain boundaries, the pressure to use in chemical potential expressions is the negative of the principal stress normal to the grain boundary, $-t_{3}$. So, for example, $(21)$ becomes

$$
\mu_{1}^{g b}=G_{1}^{s}\left(-t_{3}\right)+\frac{V_{0}}{18 \kappa}\left(q_{1}+q_{2}\right)^{2}+\frac{V_{0}}{6 \mu}\left(q_{1}^{2}-q_{1} q_{2}+q_{2}^{2}\right)
$$

in which $G_{1}^{s}$ is evaluated at $\theta$ and a pressure, $-t_{3}$, and the $q$ definitions written as $q_{1}=t_{1}-t_{3}$ and $q_{2}=t_{2}-t_{3}$. This reduces to (1) for a solid-fluid grain boundary as then $p=-t_{3}$. As the pressure, $-t_{3}$, as well as $q_{1}$ and $q_{2}$ in each solid, may vary along a solid-solid grain boundary, constant $\mu_{1}^{g b}$ along the boundary means that these variables are constrained to covary in each solid. Equation 22, and the equivalent of (19-20), apply to solid-solid grain boundaries whether the equilibrium being considered is fluid-present or not.

Use of the interface equilibrium relation to determine the chemical potentials of all additive end-members in the solids, as reflected in fluid and in grain boundaries, depends 
on the disposition of grain boundaries in the context of the way stress varies in the solids in an equilibrium. This is in contrast to considering an equilibrium involving only hydrostatically-stressed phases. Then the disposition of grain boundaries is irrelevant (if the grain size is not very small and the boundaries are essentially planar), and the chemical potentials can be calculated without consideration of spatial information.

\section{ILLUSTRATIVE CALCULATIONS}

Simple forward calculations are shown here for equilibrium of elastic solids with and without fluid. In the calculations, minerals are treated as isotropic solids, allowing the effect of non-hydrostatic stress to be illustrated in a straightforward way. As outlined in Appendices 1 and 2 , little is gained by using more complicated thermodynamic relations that include the effect of anisotropy.

First, calculations with 1-end-member solids are considered, using kyanite + sillimanite with and without fluid as an example. If the minerals are hydrostatically stressed, then equilibrium coexistence occurs on a $p-\theta$ line. If temperature is fixed, then coexistence can only occur at one pressure. If one or both minerals are non-hydrostatically-stressed, the interface equilibrium relation gives the conditions of equilibrium coexistence which is a pressure range at given $\theta$.

Consider equilibrium coexistence of kyanite and sillimanite with fluid. At a kyanite-fluid grain boundary at equilibrium using (21)

$$
\mu_{\mathrm{Al}_{2} \mathrm{SiO}_{5}}^{f}=G^{\mathrm{ky}}+\left(\frac{V}{18 \kappa}\left(q_{1}+q_{2}\right)^{2}+\frac{V}{6 \mu}\left(q_{1}^{2}-q_{1} q_{2}+q_{2}^{2}\right)\right)^{\mathrm{ky}}
$$

Similarly, at a sillimanite-fluid grain boundary

$$
\mu_{\mathrm{Al}_{2} \mathrm{SiO}_{5}}^{f}=G^{\text {sill }}+\left(\frac{V}{18 \kappa}\left(q_{1}+q_{2}\right)^{2}+\frac{V}{6 \mu}\left(q_{1}^{2}-q_{1} q_{2}+q_{2}^{2}\right)\right)^{\text {sill }}
$$

in which $V$ is $V_{0}$, the volume of the end-member at zero stress and the temperature of interest. In an equilibrium involving kyanite + sillimanite + fluid, $\mu_{\mathrm{Al}_{2} \mathrm{SiO}_{5}}^{f}$ is constant, and the fluid pressure is constant, $p$. Therefore (23) can be subtracted from (24), cancelling $\mu_{\mathrm{Al}_{2} \mathrm{SiO}_{5}}^{f}$, giving

$$
\begin{aligned}
0=G^{\text {sill }}-G^{\mathrm{ky}}+ & \left(\frac{V}{18 \kappa}\left(q_{1}+q_{2}\right)^{2}+\frac{V}{6 \mu}\left(q_{1}^{2}-q_{1} q_{2}+q_{2}^{2}\right)\right)^{\text {sill }}- \\
& \left(\frac{V}{18 \kappa}\left(q_{1}+q_{2}\right)^{2}+\frac{V}{6 \mu}\left(q_{1}^{2}-q_{1} q_{2}+q_{2}^{2}\right)\right)^{\mathrm{ky}}
\end{aligned}
$$


Linearising thermodynamic data for the kyanite $=$ sillimanite reaction leads to, $G^{\text {sill }}-G^{\mathrm{ky}} \approx 6.876-0.011384 \theta+0.5445 p \mathrm{~kJ}$, at $7 \mathrm{kbar}$ and $650^{\circ} \mathrm{C}$ (Holland \& Powell, 2011, using the units, kJ, K and kbar adopted there). The kyanite + sillimanite equilibrium coexistence surface involves $p, \theta$, and four $q$ values. The effect of some subsets of these variables on the values of the other variables at equilibrium is now shown.

Envisage that equilibration occurs at $650^{\circ} \mathrm{C}$, and that a non-hydrostatically-stressed kyanite is coexisting with hydrostatically-stressed sillimanite, as might apply if the kyanite is reacting to sillimanite as $\theta$ increases. With sillimanite hydrostatically stressed, $q_{1}^{\text {sill }}=q_{2}^{\text {sill }}=0$. Under these conditions the equilibrium relation is

$$
0=-3.631+0.5445 p-\left(\frac{V}{18 \kappa}\left(q_{1}+q_{2}\right)^{2}-\frac{V}{6 \mu}\left(q_{1}{ }^{2}-q_{1} q_{2}+q_{2}{ }^{2}\right)\right)^{\mathrm{ky}}
$$

in $\mathrm{kJ}$, with $p, q_{1}$ and $q_{2}$ in kbar. To reiterate, the principal stresses of sillimanite and of kyanite grains that are perpendicular to the solid-fluid grain boundaries, are equal in magnitude, but opposite in sign, to $p$. As sillimanite in this example is taken to be hydrostatically-stressed then sillimanite grains can be considered to have a pressure, $p$. The state of non-hydrostatic stress in the kyanite grains at kyanite-fluid grain boundaries is represented by $q_{1}^{\mathrm{ky}}$ and $q_{2}^{\mathrm{ky}}$, and generally will vary along those grain boundaries. This equation, (26), gives the conditions for equilibrium coexistence of kyanite + sillimanite, with fluid, in terms of $p, q_{1}^{\mathrm{ky}}$ and $q_{2}^{\mathrm{ky}}$, as portrayed in Figure 4.

Considering relations at different grain boundaries in a kyanite + sillimanite + fluid system at equilibrium, a grain boundary between a non-hydrostatically-stressed grain of kyanite and fluid at equilibrium can be considered using (23). Linearising thermodynamic data for kyanite leads to, $G^{\mathrm{ky}} \approx-2486.57-0.269125 \theta+4.4717 p \mathrm{~kJ}$, at $7 \mathrm{kbar}$ and $650^{\circ} \mathrm{C}$ (Holland \& Powell, 2011). With $q_{1}$ and $q_{2}$ in the range $\pm 5 \mathrm{kbar}$, the second two terms together in (23) are $<0.03 \mathrm{~kJ}$ and are always positive, using $V / \kappa=0.0019 \mathrm{~kJ} \mathrm{kbar}^{-2}$ and $V / \mu=0.0042 \mathrm{~kJ} \mathrm{kbar}^{-2}$ as in Figure 4. This is tiny compared with the pressure dependence of $G^{\mathrm{ky}}$. Because the second two terms are always positive it means that a fluid in equilibrium with a non-hydrostatically-stressed kyanite is always supersaturated in $\mathrm{Al}_{2} \mathrm{SiO}_{5}$ with respect to fluid in equilibrium with hydrostatically-stressed kyanite (a general result of Gibbs, 1906, p.197).

For the system with a specified fluid pressure, for example, with a pressure displacement of 0.01 kbar below the kyanite-sillimanite equilibrium with hydrostatically-stressed minerals, the covarying of $q_{1}$ and $q_{2}$ along kyanite-fluid grain boundaries is given by the 0.01 ellipse in Figure 4. Given that hydrostatically-stressed sillimanite can also be considered to have a pressure, the same as the fluid pressure, the covarying of $q_{1}$ and $q_{2}$ at 
sillimanite-kyanite grain boundaries is given by the same ellipse. Along kyanite-kyanite grain boundaries, the pressure at the boundary, $-t_{3}$, can vary, as well as $q_{1}$ and $q_{2}$ in each of the adjacent grains at the grain boundary. From (23), the difference between the fluid pressure and the pressure at a kyanite-kyanite grain boundary is given by

$$
p+t_{3}=\frac{0.005445-\left(\frac{V}{18 \kappa}\left(q_{1}+q_{2}\right)^{2}+\frac{V}{6 \mu}\left(q_{1}^{2}-q_{1} q_{2}+q_{2}^{2}\right)\right)^{\mathrm{ky}}}{4.4717}
$$

with $0.005445 \mathrm{~kJ}$ the $\mu_{\mathrm{Al}_{2} \mathrm{SiO}_{5}}^{f}$ difference corresponding to the displacement of the equilibrium by $0.01 \mathrm{kbar}$, and $4.4717 \mathrm{~kJ} / \mathrm{kbar}$ the pressure dependence of $G^{\mathrm{ky}}$. This pressure, $-t_{3}$, can be larger or smaller than the fluid pressure. With $q_{1}$ and $q_{2}$ in the range $\pm 5 \mathrm{kbar}, p+t_{3}$ is in the range -0.011 to $0.0012 \mathrm{kbar}$. This is a very small pressure range.

In this consideration of solid-solid grain boundaries in a fluid-present equilibrium, the pressure along the grain boundaries can be related to the fluid pressure, and is very similar to it. In the absence of fluid, the calculation can be recast to give the pressure at the grain boundaries directly. Again, the pressure range at the grain boundaries in an equilibrium is very small. The small pressure range in both these cases is due primarily to the pressure dependence of $G$ being much larger than the contribution of the $q$ terms.

If both sillimanite and kyanite are non-hydrostatically-stressed, and using the simplification for each mineral that $q_{1}=q_{2}=q$ to allow a two-dimensional representation, the surface of coexistence can be portrayed as in Figure 5. The calculations indicate again that kyanite + sillimanite coexistence can only occur over a very narrow pressure range at a fixed temperature, with the pressure range extending up and down pressure from the position of the kyanite = sillimanite univariant for both minerals hydrostatically-stressed, depending on whether the effect of the non-hydrostatic stress in kyanite dominates (up), or sillimanite (down)

A very small range of equilibrium coexistence of minerals involved in reactions that are univariant when the minerals are hydrostatically-stressed is a general result. The implication drawn is not dependent on the precise values of the moduli, $\kappa$ and $\mu$. For example, halving the moduli, $\kappa$ and $\mu$ do not invalidate the conclusion that the contribution of the $q$ terms to the thermodynamics is small. The lack of consistency of this result with Wheeler (2014) is discussed in Appendix 3.

Next, calculations with solid solutions are considered. The example considered here is that of the expected compositional heterogeneity in a mosaic of heterogeneously non-hydrostatically stressed grains of a binary plagioclase in an equilibrium. At equilibrium, $\mu_{(\mathrm{CaAl})_{-1}(\mathrm{NaSi})}$, i.e. $\mu_{\mathrm{ab} \text { an }}$, defined by $(16)$, is constant in each grain and 
between grains, with each plagioclase grain having smoothly varying mean stress. The occurence of both mean stress and composition in the activity terms in (16), means that at constant $\mu_{\mathrm{ab} \text { an }}$ the grains must be compositionally-zoned. There will be composition steps at grain boundaries corresponding to mean stress steps. Consider two points in grains within the mosaic at equilibrium, $\mathrm{A}$ and $\mathrm{B}$. The value of the exchange chemical potentials is the same everywhere, so

$$
\mu_{\mathrm{ab} \text { an }}^{\mathrm{A}}=\mu_{\mathrm{ab} \text { an }}^{\mathrm{B}}
$$

where the superscripts A and B refer to the physical locations. For clarity, plagioclase is assumed to be an ideal solid solution, and $x$ is substituted for $a_{\text {an }}$ in (16) and $1-x$ for $a_{\text {ab }}$. Simplifying, (27) becomes

$$
\mathrm{R} \theta \ln \frac{1-x^{\mathrm{A}}}{x^{\mathrm{A}}}-\overline{\mathrm{T}}^{A} V_{\mathrm{ab} \text { an }}=\mathrm{R} \theta \ln \frac{1-x^{\mathrm{B}}}{x^{\mathrm{B}}}-\overline{\mathrm{T}}^{B} V_{\mathrm{ab} \text { an }}
$$

with $V_{\mathrm{ab} a n}=V_{\mathrm{ab}}-V_{\mathrm{an}}$.

In (28), if point $\mathrm{A}$ has a composition, $x$, at a mean stress, $\overline{\mathrm{T}}$, and point $\mathrm{B}$ has the composition, $x+\Delta x$, at mean stress, $\overline{\overline{\mathrm{T}}}+\Delta \overline{\mathrm{T}}$, then rearranging (28) gives

$$
\ln \frac{(1-x)(x+\Delta x)}{x(1-x-\Delta x)}=-\Delta \overline{\mathrm{T}} \frac{V_{\mathrm{ab} \text { an }}}{\mathrm{R} \theta}
$$

A series expansion of the left-hand side for $\Delta x$ around zero gives

$$
\ln \frac{(1-x)(x+\Delta x)}{x(1-x-\Delta x)} \approx \frac{\Delta x}{x(1-x)}
$$

So, to a first approximation

$$
\Delta x=-x(1-x) \frac{\Delta \overline{\mathrm{T}} V_{\mathrm{ab} \text { an }}}{\mathrm{R} \theta}
$$

with $V_{\mathrm{ab} \text { an }}=0.039 \mathrm{~kJ} \mathrm{kbar}^{-1}$ (at $\theta=650^{\circ} \mathrm{C}$ and $8 \mathrm{kbar}$ ), and with $x=0.3, \theta=650^{\circ} \mathrm{C}$ and $\Delta \overline{\mathrm{T}}=3.3 \mathrm{kbar}, \Delta x \approx-0.0035$. Such a composition difference is similar to the random error associated with electron microprobe analysis of plagioclase composition. The difference between this result and that of Tajčmanová, Podladchikov, Powell, Moussas, Vrijmoed and Connolly (2014) is discussed in Appendix 4.

The plagioclase $V_{\mathrm{ab} \text { an }}$ in (29) is relatively small, but such a $V$ tends to be small for the majority of substitutions in minerals where there is significant substitution, so that large $q$ tending to give rise to very small composition changes is a general result. Equation 29 is a general expression for the effect of variation in $\overline{\mathrm{T}}$ on an exchange equilibrium. The same 
logic as in (29) applies if there is more than one exchange operating in a mineral. If there are several minerals in the equilibrium with the same exchange, say $(\mathrm{CaAl})_{-1}(\mathrm{NaSi})$, $\mathrm{Na}_{-1} \mathrm{~K}$, or $\mathrm{Mg}_{-1} \mathrm{Fe}$, then (29) applies in each of them. Given that the composition variation due to mean stress variation is so small, if minerals have obvious zoning then it is unlikely to be because the minerals are non-hydrostatically stressed, unless the $V$ term for the exchange is much larger than for plagioclase.

Continuing with this binary plagioclase example, consider now a plagioclase-fluid grain boundary. Application of the interface equilibrium relation, using $(19,20)$, and the assumption that plagioclase is an ideal solid solution, as above, gives

$$
\begin{gathered}
\mu_{\mathrm{an}}^{f}=G_{\mathrm{an}}(p)+\mathrm{R} \theta \ln (1-x)+\frac{1}{3} x\left(q_{1}+q_{2}\right) V_{\mathrm{ab} \text { an }}+ \\
\frac{V_{0}}{18 \kappa}\left(q_{1}+q_{2}\right)^{2}+\frac{V_{0}}{6 \mu}\left(q_{1}^{2}-q_{1} q_{2}+q_{2}^{2}\right) \\
\mu_{\mathrm{ab}}^{f}=G_{\mathrm{ab}}(p)+\mathrm{R} \theta \ln x-\frac{1}{3}(1-x)\left(q_{1}+q_{2}\right) V_{\mathrm{ab} \text { an }}+ \\
\frac{V_{0}}{18 \kappa}\left(q_{1}+q_{2}\right)^{2}+\frac{V_{0}}{6 \mu}\left(q_{1}^{2}-q_{1} q_{2}+q_{2}^{2}\right)
\end{gathered}
$$

in which $V_{0}$ is the volume of plagioclase, unstressed, at the temperature and composition of interest. The $\mu^{f}$ values are the ones that would be used to calculate conditions of equilibrium with the end-members of other minerals coexisting with the plagioclase. The contribution to $\mu$ from non-hydrostatic stress, given by the last three terms in the equations, can be compared with the uncertainties on end-member chemical potentials given in thermodynamic data compilations. The uncertainties in $G_{\mathrm{ab}}$ and $G_{\mathrm{an}}$ are 3.36 and $1.58 \mathrm{~kJ}$, respectively, $\left(2 \sigma_{\Delta_{\mathrm{f}} H}\right.$, Holland \& Powell, 2011). Adding the latter three terms in the $\mu^{f}$ expressions, with $V=10 \mathrm{~kJ} \mathrm{kbar}^{-1}, \kappa=600 \mathrm{kbar}, \mu=360 \mathrm{kbar}$, and $q_{1}$ and $q_{2}$ in the range $\pm 5 \mathrm{kbar}$, gives $<0.35 \mathrm{~kJ}$, small compared to the dataset uncertainties. The covarying relationship amongst $x, q_{1}$ and $q_{2}$ along plagioclase-fluid grain boundaries can be found from (29) and the mean stress definition. Specifying the fluid to be at $p=8 \mathrm{kbar}$, and the plagioclase at a point on the grain boundary to have $x=0.3$ and $q_{1}=q_{2}=-3$ kbar (giving $\overline{\mathrm{T}}=-10 \mathrm{kbar}$ ), then, by (29), $\Delta x=-0.0011 \overline{\mathrm{T}}$. Then, from the mean stress definition, $q_{2}=-q_{1}+3 \Delta \overline{\mathrm{T}}-6$. The logic for plagioclase-plagioclase grain boundaries follows the kyanite example above, with $-t_{3}$ being in a small range around $p$.

Can it be recognised if solid solutions were non-hydrostatically stressed at the time that textures and mineral compositions were preserved? The calculations above suggest that it 
might be difficult to do. Patterns of composition steps at grain boundaries might feasibly help, but, in a rock context, diffusive equilibration may change composition relations at grain boundaries after the assemblage as a whole has stopped evolving. The best opportunity might be provided by composition variation within grains of solid that involve exchanges with the largest $V_{i j}$, which are likely to be ones involving more limited substitutions. Calculations relating to such effects would need to involve a full exposition of the thermodynamics, involving less simplified compliance tensors for minerals than those used for illustrative purposes here, including $p, \theta$ and composition dependence of the moduli involved.

An important difference in calculation methods between systems involving just hydrostatically-stressed minerals, and systems involving non-hydrostatically-stressed minerals, relates to the possibility of using straightforward energy minimisation techniques. When all minerals are assumed to be hydrostatically stressed, Gibbs energy minimisation is good for phase equilibrium calculations, as for example done by Perplex (Connolly 2009). However, in non-hydrostatically stressed systems this is not an option. As shown by the original energy minimisation in Larché and Cahn (1973), such a minimisation is non-trivial, requiring the variational calculus. Given the heterogeneity of stress in general, there is no direct way to calculate mineral proportions and compositions using energy minimisation.

The above illustrative calculations on non-hydrostatically-stressed minerals involve forward calculations. In principle, inverse chemical potential-based calculation methods, like average pressure (avp, Powell \& Holland, 1994), can also be undertaken. Average pressure can be calculated based on observed mineral compositions if the states of stress are known in each mineral. Generally in rocks the states of stress are not known, and an inverse method that specifically accounted for these would not be constrained. However, chemical potentials, as in (30-31), and mineral composition, as in (29), are very insensitive to the state of stress. Consequently, in applying inverse methods such as average pressure, it is sufficient to assume that the minerals are hydrostatically-stressed.

Regardless of the actual or assumed state of stress in the minerals, the average pressure will refer to the average of the pressure along the grain boundaries, and the fluid pressure if the equilibrium is fluid-present. This is because it is the interface equilibrium relation for the end-members in each mineral that specifies the chemical potentials at grain boundaries (and in fluid if there is one). These are the chemical potentials used in the $\Delta \mu=0$ relations that are then combined in an avp calculation. The mean stress in the minerals is little reflected in the calculated average pressure.

The small difference between the thermodynamics of minerals in a 
non-hydrostatically-stressed state and a corresponding hydrostatic state suggests that various conventional thermodynamic calculations that assume hydrostatically-stressed minerals will give useful results even if the minerals are non-hydrostatically-stressed. This result provides an a postiori justification for the adoption by Bruton and Helgeson (1983) and Dahlen (1992) of their more restrictive approximation: making $F+p V$ in (17) independent of stress, which, in terms of the formulation above means that $V_{0} / \kappa$ and $V_{0} / \mu$ are both taken to be zero, e.g. in (21).

\section{DISCUSSION and CONCLUSIONS}

As noted in the Introduction, the systematic nature of observations on metamorphic mineral assemblages is consistent with a local equilibrium interpretation of what is seen. The observations suggest a priori that non-hydrostatic stress in minerals does not have a first order effect. The illustrative calculations using the thermodynamic development above show that caleulations that incorporate the equilibrium thermodynamics of non-hydrostatically-stressed minerals give results that are little different from those assuming hydrostatic stress. Making the usual assumption that, to first order, mineral assemblages as preserved in rocks do represent equilibria on an appropriate length-scale, the equilibrium methods developed above become directly applicable to rocks. Thus, the presence of non-hydrostatically stressed minerals in rocks at the time that equilibrium is preserved is unlikely to significantly affect the results of approaches commonly adopted in mineral equilibria calculations, like thermobarometry, that involve the implicit assumption that the minerals are hydrostatically stressed. The a posteriori success of such approaches, along with their ability to account for the fundamental patterns of mineral assemblage occurrence in orogens, is consistent with this. A view of metamorphism in which non-hydrostatic effects dominate, as in Wheeler (2014), is inconsistent with both the thermodynamic approach outlined here and the rock record itself.

Mineral assemblage evolution during progressive metamorphism, prior to mineral assemblage preservation, accompanies elasto-viscoplastic processes accumulating permanent deformation. The dissipative processes that need to take place to keep establishing an approach to equilibrium appear to take place readily as pressure-temperature conditions change. Such an approach to equilibrium is a necessary implication of many of the observations on mineral assemblages that result from preservation. While dissipative processes may leave no mark on the final preserved inferred-equilibrium mineral assemblage, it may leave a record in features such as prograde-zoned porphyroblasts. 
In general, overprinting equilibration is more complicated if the minerals are non-hydrostatically stressed. At equilibrium, if all the minerals are hydrostatically stressed, they are homogeneous and can be considered to have a pressure, the same in all of the minerals, and equal to that in a fluid. As a consequence this pressure can be treated as a boundary condition of the local equilibrium. This pressure might then be related to the depth of metamorphism, or be understood in terms of larger scale tectonic processes. The imposition of a pressure (and temperature) boundary condition is what allows Gibbs energy minimisation to work for mineral equilibrium calculations. Boundary conditions for elastic systems are, however, a more difficult problem, as discussed by, for example, Truesdell and Noll (2004, p125 et seq.), and McLellan (1980, sections 19.3 and 22.4). In the elastic case, the lack of homogeneity of stress-strain and the relevance of grain boundaries means that the relation between boundary conditions and the behaviour of a local equilibrium is indirect (e.g. Dahlen, 1992). In general, the connection can only be made via the variational calculus (e.g. Larché \& Cahn, 1973, 1978), and solution of the appropriate partial differential equations. Detailed consideration of the processes involved in equilibration itself is beyond our remit here.

The emphasis above is on equilibrium stated in terms of chemical potentials of the end-members of minerals because that is the form that the variational calculus gives. The approach of, for example, Powell and Holland (1994) and Powell, Holland and Worley (1998), which is focussed on equilibrium in terms of the equilibrium relations, $\Delta \mu=0$, for balanced reactions between end-members, carries across to handling systems involving non-hydrostatically stressed minerals, e.g. via (19).

There remain questions regarding how mineral assemblage development in progressive metamorphism is curtailed and the resulting mineral assemblage preserved. The loss of fluid around the start of cooling, Guiraud et al. (2001), or the loss of melt at higher metamorphic grades, White and Powell (2002), is certainly significant. We speculate that an additional effect comes from the evolution of elastic behaviour and chemical equilibration during cooling, following elasto-viscoplastic behaviour giving way to elastic behaviour.

\section{ACKNOWLEDGEMENTS}

RP acknowledges those several people who sent him Wheeler (2014) when it first came online (knowing that he wouldn't have seen it otherwise). He thanks Lucie Tajc̆manová for her support in visiting ETH Zürich where, through conversations with Lucie and Yuri 
Podladchikov, the original motivation for this work came. We thank Jamie Connolly and Bruce Hobbs for discussions and the former for checking his elastic energy calculation in Connolly (2009). We thank Bruce Hobbs (x2), John Wheeler, Ross Angel, Michel Guiraud and three anonymous reviewers for their reviews of earlier versions of the paper. Editor, Mike Brown, is thanked for his perseverance and patience.

\section{REFERENCES}

Alberty, R.A. (2001). Use of Legendre transforms in chemical thermodynamics. Pure and Applied Chemistry, 73(8), 1349-1380.

Balluffi, R.W., Allen, S.M., \& Carter, W.C. (2005). Kinetics of Materials. John Wiley \& Sons, New York, 644pp.

Brown, J.M., Angel, R.J., \& Ross, N. (2016). Elasticity of plagioclase feldspars. Journal of Geophysical Research, Solid earth, 121(2), 663-675.

Cahn, J., \& Larché, F.C. (1983). An invariant formulation of multicomponent diffusion in crystals. Scripta Metallurgica, 17(7), 927-932.

Callen, H.B. (1985). Thermodynamics and introduction to thermostatistics. John Wiley \& Sons, New York, 493pp.

Cermelli, P., \& Gurtin, M.E. (1994). The dynamics of solid-solid phase transitions. 2. Incoherent interfaces. Archive for Rational Mechanics and Analysis, 127(1), 41-99.

Coble, R.L. (1963). A model for boundary diffusion controlled creep in polycrystalline materials. Journal of Applied Physics, 34 (6), 1679-1682.

Connolly, J.A.D. (2009). The geodynamic equation of state. What and how. G-cubed, $10(10), 1-19$.

Dabrowski, M, Powell, R \& Podladchikov, Y (2015). Viscous relaxation of grain-scale pressure variations. Journal of Metamorphic Geology, 33(8), 859-868.

Dahlen, F.A. (1992). Metamorphism of non-hydrostatically stressed rock. American Journal of Science, 292(3), 184-198.

Darken, L.S. (1942). Diffusion in metal accompanied by phase change. Transactions of AIME, $175(2), 157-171$. 
Dealy, J.M. (2010). Weissenberg and Deborah numbers - their definition and use. Rheology Bulletin, 10(2), 14-17.

Frolov, T., \& Mishin, Y. (2010). Effect of non-hydrostatic stresses on solid fluid equilibrium. Bulk thermodynamics. Physical Review B, 82(14), 174113.

Fung, Y.C., \& Tong, P. (2001). Classical and computational continuum mechanics. World Scientific Publishing, Singapore. 951pp.

Fyfe, W.S, Turner, F.J., \& Verhoogen, J. (1958). Metamorphic reactions and metamorphic facies. Geological Society of America, Memoir, 73, 258pp.

Gibbs, J.W.(1906). The Scientific Papers: Thermodynamics. Longmans, Green and co, London, 434 pp.

Guggenheim, E.A.(1967). Thermodynamics. Elsevier, 390pp.

Guiraud, M., Powell, R., \& Rebay, G. (2001). $\mathrm{H}_{2} \mathrm{O}$ in metamorphism and unexpected behaviour in the preservation of metamorphic mineral assemblages, Journal of Metamorphic Geology, 19(4), 445-454.

Gurtin, M.E., Fried, E. \& Anand, L. (2010). The mechanics and thermodynamics of continua. Cambridge University Press, New York. 694pp.

Herring, C. (1950). Diffusional viscosity of a polycrystalline solid. Journal of Applied Physics, 21(5), 437-445.

Hobbs, B.E., \& Ord, A. (2015). Structural geology: the mechanics of deforming metamorphic rocks, Elsevier, 665pp.

Hobbs, B.E., \& Ord, A. (2016). Does non-hydrostatic stress influence the equilibrium of metamorphic reactions. Earth Science Reviews, 163, 190-233.

Holland, T.J.B., \& Powell, R. (1992). Plagioclase feldspars: activity-composition relations based upon Darken's quadratic formalism and Landau theory. American Mineralogist, $77(1), 53-61$.

Holland, T.J.B., \& Powell, R. (2011). An improved and extended internally-consistent thermodynamic dataset for phases of petrological interest, involving a new equation of state for solids. Journal of Metamorphic Geology, 29(3), 333-383. 
Johnson, W.C. \& Schmalzried, H. (1992). Gibbs-Duhem and Clausius-Clapeyron type equations for elastically-stressed crystals. Acta Metallurgica et Materialia, 40(9), $2337-2342$.

Kamb, W.B. (1961). The thermodynamic theory of non-hydrostatically stressed solids. Journal of Geophysical Research, 66(1) 259-271.

Kirkwood, J.G. \& Oppenheim, I. (1961). Chemical thermodynamics. McGraw-Hill, New York. 261pp.

Korzhinskii,D.S. (1959). Physicochemical basis of the analysis of the paragenesis of minerals, (translated from the Russian by Consultants Bureau, Inc, New York, 142pp

Lai, M., Rubin, D., \& Krempl E. (2010). Introduction to continuum mechanics. Elsevier, Amsterdam.520pp.

Larché, F.C. \& Cahn, J.W. (1973). A linear theory of thermochemical equilibrium under stress. Acta Metallurgica, 21(8), 1051-1063.

Larché, F.C. \& Cahn, J.W. (1978). Thermochemical equilibrium of multiphase solids under stress. Acta Metallurgica, 26 (10), 1579-1589.

Larché, F.C. \& Cahn, J.W. (1985). The interactions of composition and stress in crystalline solids. Acta Metallurgica, 33(3), 331-357.

Leo, P.H. \& Sekerka, R.F. (1989). The effect of surface stress on crystal-melt and crystal-crystal equilibrium. Acta Metallurgica, 37(12), 3119-3138.

Li, J.C.M, Oriani, R.A., \& Darken, L.S. (1966). The thermodynamics of stressed solids. Zeitschrift für Physikalische Chemie Neue Folge, 49(3/4), 271-290.

Llana-Fúnez, S., Wheeler, J, \& Faulkner, D.R. (2012). Metamorphic reaction rate controlled by fluid pressure not confining pressure: implications of dehydration experiments with gypsum. Contributions to Mineralogy and Petrology, 164(1), 69-79.

McLellan, A.G. (1980). The classical thermodynamics of deformable media. Cambridge University Press, Cambridge. 338pp.

Malvern, L.E. (1969). Introduction to the mechanics of a continuous medium. Prentice-Hall, New Jersey. 713pp. 
Mishin, Y., Warren, J.A., Sekerka, J.F., \& Boettinger, W.J. (2013). Irreversible thermodynamics of diffusion creep in crystalline solids. Physical Review B, 88(18), 184303.

Mullins, W.W., \& Sekerka, R.F. (1985). On the thermodynamics of crystalline solids. Journal of Chemical Physics, 82(11), 5192-5202.

Münster, A. (1970). Classical thermodynamics. John Wiley \& Sons, London. 387pp.

Mura, T. (1987). Micromechanics of defects in solids. 2nd ed. Kluwer, Dordercht. 587pp.

Nye, J.F. (1985), Physical properties of crystals. Oxford University Press, Oxford. 329 pp.

Paterson, M.S. (1973). Nonhydrostatic thermodynamics and its geologic applications. Reviews of Geophysics and Space Physics, 11(2), 355-389.

Paterson, M.S. (2013). Material science for structural geology. Springer, Dordrecht. $254 \mathrm{pp}$

Powell, R, \& Holland, T.J.B. (1994). Optimal geothermometry and geobarometry. American Mineralogist, $79(1 / 2), 120-133$.

Powell, R., Holland, T.J.B., \& Worley, B. (1998). Calculating phase diagrams involving solid solutions via non-linear equations, with examples using THERMOCALC Journal of Metamorphic Geology, 16(4), 577-588.

Powell, R., White, R.W., Green, E.C.R., Holland, T.J.B., Diener, J.F.A.. (2014). On parameterising activity-composition relations of minerals for petrological calculations. Journal of Metamorphic Geology, 32(3), 245-260.

Reiner, M. (1964). The Deborah number. Physics Today, 17(1), 62.

Rutter, E.H., \& Elliot, D. (1976). The kinetics of rock deformation by pressure solution. Philosphical Transactions of the Royal Society, London, A, 283(1312), 203-219.

Sekerka, R.F., \& Cahn, J.W. (2004). Solid-liquid equilibrium for non-hydrostatic stress. Acta Materialia, 52(6), 1663-1668.

de Souza Neto, E.A., Perić, D., \& Owen, D.R.J. (2008). Computational methods for plasticity. Wiley \& Sons (Chichester, UK), 791pp.

S̆típská, P, Powell, R, White, RW, \& Baldwin, JA (2010). Using calculated chemical potential relationships to account for coronas around kyanite: an example from the Bohemian Massif. Journal of Metamorphic Geology, 28(1), 97-116. 
Tadmor, E.B., Miller, R.E. \& Elliot (2012). Continuum Mechanics and thermodynamics. Cambridge University Press, New York. 350pp.

Tajčmanová, L., Poddatchikov, Y.Y., Powell, R., Moulas, E., Vrijmoed, J.C. \& Connolly, J.A.D. (2014). Grain scale pressure variations and chemical equilibrium in high-grade metamorphic rocks. Journal of Metamorphic Geology, 32(2), 195-207.

Tajčmanová, L., Moulas, E., \& Vrijmoed, J.C. (2015). Grain-scale pressure variations in metamorphic rocks: implications for the interpretation of petrographic observations. Lithos, 216-217(2), 338-351.

Thompson, J.B. (1955). The thermodynamic basis for the mineral facies concept. American Journal of Science, 253(2), 65-103.

Thompson, J.B. (1959). Local equilibrium in metasomatic processes. In PH Abelson (ed.), Researches in Geochemistry, 2, John Wiley \& Sons, 427-457.

Truesdell, C, \& Noll, W. (2004). The non-linear field theories of classical mechanics (3ed. ed. S.S. Antman). Springer-Verlag, Heidelberg. 627pp.

Wheeler, J. (2014). Dramatic effects of stress on metamorphic reactions. Geology 42(8), 647-650.

White, R.W., \& Powell, R. (2002). Melt loss and the preservation of granulite facies mineral assemblages. Journal of Metamorphic Geology, 20(7), 621-632.

White, R.W., Powell, R., Holland, T.J.B., Johnson, T.E., \& Green E.C.R. (2014). New mineral activity-composition relations for thermodynamic calculations in metapelitic systems. Journal of Metamorphic Geology, 32(3), 261-286.

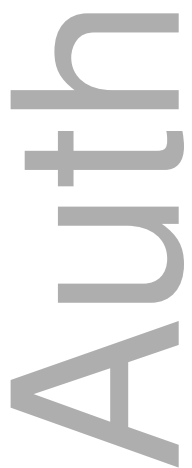




\section{APPENDIX 1: APPROXIMATION FOR CHEMICAL POTENTIALS OF EXCHANGE END-MEMBERS}

To use the general equation, (15), for calculations, approximations are needed. Following Larché and Cahn (1973), p1059, and Larché and Cahn (1985), p338, adopting isotropic constitutive relations, the eigenstrain is spherical. Then $V_{\ominus} \mathbf{E}_{\mathbf{c}}$ can be approximated for small eigenstrains (the first approximation in the next equation) by $\left(V-V_{\ominus}\right) \mathbf{1}$, involving the volume, $V$, at $\theta$ in the unstressed state and at the composition of interest. At this point, $\partial \mathbb{S} / \partial n_{12}=0$ is also assumed. Then

$$
\mu_{12}(\mathbf{T})-\left(\mu_{12}\right)_{\ominus} \approx-\frac{1}{3}(\mathbf{1}: \mathbf{T})\left(\frac{\partial V}{\partial n_{12}}\right) \approx-\overline{\mathrm{T}}\left(V_{1}-V_{2}\right) \equiv-\overline{\mathrm{T}} V_{12}
$$

with $V_{12}$ defined as the difference in the molar volumes of pure end-member 1 and 2, $V_{1}-V_{2}$, at $\theta$ in the unstressed state, and in the absence of an excess volume of mixing (the second approximation). The assumptions leading to (32) result in the dependence of $\mu_{12}(\mathbf{T})$ on $\mathbf{T}$ reducing to a dependence on the first principal invariant of $\mathbf{T}$, i.e. the mean stress, $\overline{\mathbf{T}}$.

Now to determine $\left(\mu_{12}\right)_{\theta}$. Using a standard state of pure end-member at the $\theta$ of interest and an arbitrary pressure, $P$, denoted by superscript 0 , and noting that the approximation $\partial \mathbb{S} / \partial n_{12}=0$ means that the moduli in the compliance, $\mathbb{S}$, are composition independent, then, $\left(\mu_{12}\right)_{\ominus}$ in $(32)$ can be written

$$
\left(\mu_{12}\right)_{\ominus}=\mu_{1}^{0}(0)-\mu_{2}^{0}(0)=\mu_{1}^{0}(P)-\mu_{2}^{0}(P)-P V_{12}
$$

From $(32,33)$, the chemical potential, $\mu_{12}(\mathbf{T})$, which is for the stressed state, can now be written in terms of the chemical potentials of the additive end-members, [4.23]

$$
\mu_{12}(\mathbf{T})=\mu_{1}^{0}(P)-\mu_{2}^{0}(P)+\mathrm{R} \theta \ln \frac{a_{1}}{a_{2}}-V_{12}(P+\overline{\mathrm{T}})
$$

The various assumptions involved in this appendix can be relaxed at the cost of a much more complicated equation to replace (34). Generally, the added complexity involves only a minor modification of the effect of (34). However the assumption that the moduli are composition-independent is not a good approximation for some minerals, for example in plagioclase $\kappa$ is strongly composition dependent (Brown, Angel \& Ross, 2016). Treating plagioclase as isotropic, and with a composition dependent $\kappa, V_{\mathrm{ab}}$ an in (29) is modified by a term $\frac{2 \bar{T}}{\kappa^{2}} \frac{d \kappa}{d x}$. Even in this extreme case, this additional term is about half an order of magnitude less than $V_{\mathrm{ab} \text { an }}$, so, while significant, it has no effect on the implications drawn from application of (29). 


\section{APPENDIX 2: A USEFUL FORM FOR $F+p V$ IN THE INTERFACE EQUILIBRIUM RELATION}

The Helmholz energy is required for the interface equilibrium relation. The derivation, which follows Larché and Cahn (1985) closely, needs to start with the Gibbs energy, because its differential has stress, not strain, as the natural variable. As a consequence, integration with respect to stress can be undertaken with the Gibbs energy but not the Helmholz energy. The energies initially will be energy densities, with respect to a reference volume, $V_{0}$ (primed quantities in Larché \& Cahn, 1985), with $V_{0}$ being the volume of the unstressed solid at the temperature and composition of interest. Lower case is used for such densities. The differential of the Gibbs energy density for a elastic solid, [4.12] (Larché \& Cahn, 1985, with numbers in square brackets being their equations), from (11)

$$
d g=-s d \theta-\mathbf{E}: d \mathbf{T}+\sum_{i \neq \ell}^{n} \mu_{i \ell} d \rho_{i}
$$

in which the $\rho_{i}$ are number densities, with $n_{i}=V_{0} \rho_{i}$. For a 1-end-member solid, the sum disappears. This differential can be integrated from an arbitrary pressure $P$ to $\mathbf{T}$ (where $P$ will in due course be the pressure of a coexisting fluid $p$ in the interface equilibrium relation), [4.24]

$$
g(\mathbf{T})-g(P)=-\frac{1}{2} \mathbb{S} \mathbf{T}: \mathbf{T}+\left.\frac{1}{2}(\mathbb{S T}: \mathbf{T})\right|_{\mathbf{T}=-P \mathbf{1}}=-\frac{1}{2} \mathbb{S} \mathbf{T}: \mathbf{T}+\frac{1}{2} P^{2} \mathbb{S} \mathbf{1}: \mathbf{1}
$$

with the energies indicated to be a function of stress (or pressure), and considering the solid to be linear elastic, so $\mathbf{E}=\mathbb{S T}$. Using the Legendre transform [4.11], $g=f-\mathbf{E}: \mathbf{T}$, for $g(\mathbf{T})$ and also for $g(P)$ in (35) and rearranging gives, [4.25]

$$
f(\mathbf{T})=f(P)+\frac{1}{2} \mathbb{S} \mathbf{T}: \mathbf{T}-\frac{1}{2} P^{2} \mathbb{S} \mathbf{1}: \mathbf{1}
$$

Given that, for a hydrostatically-stressed solid, [4.26]

$$
f(P)=\frac{G(P)}{V_{0}}-P \frac{\left.V\right|_{\mathbf{T}=-P \mathbf{1}}}{V_{0}}
$$

the Helmholz energy density may be calculated from tabulated data of the molar Gibbs energy as a function of $P$ at specified $\theta$ and composition. This $G$ relates to $\mathcal{L}_{p \theta} U$, whereas (35) relates to $\mathcal{L}_{\mathbf{T} \theta} U$. Ultimately the $G(P)$ in (37) will be written as $\sum c_{k} \mu_{k}(p)$, in which $c_{k}$ is the proportion of end-member, $k$. In the case of a 1-end-member solid, $G(P)$ is just 
the Gibbs energy of the end-member. Now, given that the last term on the right-hand side of (37) is $P(1-P \mathbb{S} \mathbf{1}: \mathbf{1})$, then (36) becomes, [4.29]

$$
f(\mathbf{T})=\frac{G(P)}{V_{0}}+\frac{1}{2} \mathbb{S} \mathbf{T}: \mathbf{T}-P\left(1-\frac{P}{2} \mathbb{S} \mathbf{1}: \mathbf{1}\right)
$$

In this equation, for an isotropic solid, [4.30], aligning the coordinates with the principal axes of $\mathbf{T}$, and with $\left\{t_{1}, t_{2}, t_{3}\right\}$ along the diagonal of $\mathbf{T}$

$$
\frac{1}{2} \mathbb{S} \mathbf{T}: \mathbf{T}=\frac{1}{18 \kappa}\left(t_{1}+t_{2}+t_{3}\right)^{2}+\frac{1}{6 \mu}\left(t_{1}^{2}+t_{2}^{2}+t_{3}^{2}-t_{2} t_{3}-t_{1} t_{2}-t_{1} t_{3}\right)
$$

and

$$
P\left(1-\frac{P}{2} \mathbb{S} \mathbf{1}: \mathbf{1}\right)=P\left(1-\frac{P}{2 \kappa}\right)
$$

assuming that $\kappa$ and $\mu$ are constants.

Now, the focus will shift to the Helmholz energy of the solid at a solid-fluid grain boundary as needed for the interface equilibrium condition. Then the principal stress normal to the interface can be set to the pressure in the fluid, $p$. This principal stress is chosen to be $t_{3}$, so $p=-t_{3}$ (compression negative), without implication for the relative magnitudes of $t_{1}, t_{2}$ and $t_{3}$. Now, $P$ becomes the pressure in the fluid, $P=p$, and $\bar{T}$ is used for the mean stress in the solid at the interface. The development of Sekerka and Cahn (2004) is brought in here, in parallel to that of Larché and Cahn (1985), with single numbers in square brackets being Sekerka and Cahn (2004) equations. In the following equations the only thing that relates to the fluid is its pressure, $p$. For an isotropic solid (38) can now be written

$$
f(\mathbf{T})=\frac{G(p)}{V_{0}}+\frac{1}{2 \kappa}(\overline{\mathrm{T}})^{2}+\frac{1}{6 \mu}\left(q_{1}^{2}-q_{1} q_{2}+q_{2}^{2}\right)-p\left(1-\frac{p}{2 \kappa}\right)
$$

with $q_{1}=t_{1}-t_{3}$ and $q_{2}=t_{2}-t_{3}$, so that when the solid is hydrostatically stressed $q_{1}=q_{2}=0$.

Now including the volume term needed in the interface equilibrium relation, forming $f(\mathbf{T})+p v(\mathbf{T})$, the additional term, $p v(\mathbf{T})$, is [35]

$$
p v(\mathbf{T})=p(1+\mathbf{E}: \mathbf{1})=p(1+\mathbb{S} \mathbf{T}: \mathbf{1})=p\left(1+\frac{\overline{\mathrm{T}}}{\kappa}\right)
$$

Combining (39) and (40), and after some algebra, using $\overline{\mathrm{T}}=-p+\frac{1}{3}\left(q_{1}+q_{2}\right)$

$$
f(\mathbf{T})+p v(\mathbf{T})=\frac{G(p)}{V_{0}}+\frac{1}{18 \kappa}\left(q_{1}+q_{2}\right)^{2}+\frac{1}{6 \mu}\left(q_{1}^{2}-q_{1} q_{2}+q_{2}^{2}\right)
$$


or in molar form

$$
F(\mathbf{T})+p V(\mathbf{T})=G(p)+\frac{V_{0}}{18 \kappa}\left(q_{1}+q_{2}\right)^{2}+\frac{V_{0}}{6 \mu}\left(q_{1}^{2}-q_{1} q_{2}+q_{2}^{2}\right)
$$

This equation is consistent with [6.4]. Also, as the two last terms are equal to zero for hydrostatically-stressed solids, with $q_{1}=q_{2}=0$, then $F+p V=G$ as expected.

Also from (39-41), the elastic energy density in the solid, $\frac{1}{2} \mathbb{S T}: \mathbf{T}$, at a solid-fluid grain boundary with a fluid at $p$, can be written

$$
\frac{p^{2}}{2 \kappa}-\frac{p}{3 \kappa}\left(q_{1}+q_{2}\right)+\frac{1}{18 \kappa}\left(q_{1}+q_{2}\right)^{2}+\frac{1}{6 \mu}\left(q_{1}^{2}-q_{1} q_{2}+q_{2}^{2}\right)
$$

which is quadratic in $p, q_{1}$ and $q_{2}$. This reduces to $p^{2} /(2 \kappa)$ when the solid is hydrostatically stressed. For kyanite at $p=8 \mathrm{kbar}$, with $\kappa=1600 \mathrm{kbar}$ and $\mu=1050 \mathrm{kbar}$, and $q_{1}$ and $q_{2}$ in the range \pm 5 , the elastic energy density is $<0.032 \mathrm{kbar}$. Such energy densities are small, for example in the context of the dataset enthalpy uncertainty, $2 \sigma_{\mathrm{H}} / V_{0}=0.3 \mathrm{kbar}$ (see also Connolly, 2009). For anisotropic solids, the number of $q$ terms in (41) and (42) depends on the crystal class. For example there are 9 independent moduli in the orthorhombic class, so 9 terms in such equations, compared with the two, involving $\kappa$ and $\mu$, for isotropic solids. The $q$ terms in (41) are always quadratic in $q_{1}$ and $q_{2}$. The elastic energy density is always small for minerals regardless of crystal class.

\section{Appendix 3: Discussion of Wheeler (2014)}

Wheeler (2014) proposes that the effect of non-hydrostatic stress on mineral equilibria is much bigger than that suggested in the present work. The aim here is to show why the conclusions of the two studies are different.

An example used by Wheeler is the breakdown of muscovite and quartz to K-feldspar, sillimanite and $\mathrm{H}_{2} \mathrm{O}$ in the simple KASH system.

$$
\mathrm{mu}+\mathrm{q} \rightarrow \mathrm{ksp}+\mathrm{sill}+\mathrm{H}_{2} \mathrm{O}
$$

Using (21) for each of the solids, equilibrium coexistence of mu $+\mathrm{q}+\mathrm{ksp}+$ sill $+\mathrm{H}_{2} \mathrm{O}$ at $650^{\circ} \mathrm{C}$, is given by

$$
0=-6.50+1.92 p+\sum r_{\ell}\left(\frac{V}{18 \kappa}\left(q_{1}+q_{2}\right)^{2}+\frac{V}{6 \mu}\left(q_{1}^{2}-q_{1} q_{2}+q_{2}^{2}\right)\right)^{\ell}
$$

in $\mathrm{kJ}$, with the sum being over the non-hydrostatically-stressed solids involved, and $r_{i}$ the reaction coefficients. The leading terms come from the thermodynamic data of Holland and 
Powell (2011), linearised at $650^{\circ} \mathrm{C}$ and $3.5 \mathrm{kbar}$, with a $2 \sigma$ uncertainty on the constant term of $0.28 \mathrm{~kJ}$, propagating to an uncertainty on pressure of the reaction at $650^{\circ} \mathrm{C}$ of 0.14 kbar. As with ky+sill coexistence in the main text, there will be a very small range of $p$ of coexistence of $\mathrm{mu}+\mathrm{q}+\mathrm{kspar}+\mathrm{ky}+\mathrm{q}+\mathrm{H}_{2} \mathrm{O}$ at specified $\theta$, very close to the pressure for this KASH univariant at specified $\theta$. For example, with quartz the only mineral non-hydrostatically-stressed in this equilibrium, and $q_{1}=q_{2}=5 \mathrm{kbar}$ for the quartz, the last term is just $-0.042 \mathrm{~kJ}$, much smaller than the enthalpy uncertainty on the reaction.

Wheeler (2014) is not concerned with equilibrium coexistence of the solids with fluid in reaction $\mathrm{A}$, but rather with the temperature at which the $\mathrm{mu}+\mathrm{q}$ breakdown reaction might occur, as shown in his fig. 2b. He is considering a system at a point in time in a dynamic dissipative process. He calculates a big range of temperature for the breakdown reaction by combining chemical potentials derived from spatially-separated minerals with different states of non-hydrostatic stress at solid-fluid grain boundaries. Wheeler uses an adaption of the interface equilibrium relation, $F+p V=\mu_{1}^{f}$, as used in deriving (17). He replaces $p$ by the principal stress normal to the grain boundary of interest, $-t_{3}$ in the present work, giving $F-t_{3} V=\mu_{1}^{f}$, as implied by (22). This is legitimate given that the equality, $p=-t_{3}$, relates to mechanical equilibrium. But as a consequence his combination of the different chemical potentials relates to fluid on grain boundaries at different fluid pressures. Yet, as pointed out in the main body of the text, the fluid pressure is constant in an equilibrium. The argument may be extended to solid-solid grain boundaries, given that at equilibrium the pressure $\left(-t_{3}\right)$ at such grain boundaries is close to the fluid pressure, as discussed in the main body of the text. Wheeler's pressure differences are possible if the chemical potentials being combined do not all arise in the same equilibrium. The scenario considered by Wheeler should be envisaged as a set of spatially-separate smaller systems involving different equilibria, with chemical potential and pressure gradients between the systems. Such pressure and chemical potential gradients help to drive the dynamic dissipative process which will ultimately give rise to larger equilibrated systems by coalescence of the smaller systems. Only when this stage has been reached could Wheeler consider the equilibrium coexistence of the spatially-separated minerals participating in reaction $\mathrm{A}$. If all the minerals occur together in equilibrium with the fluid on an appropriate length-scale, the fluid will have just one pressure, chemical potentials will be constant, and (43) will describe the equilibrium coexistence. Wheeler's calculations attempt to reflect the details of what has been consumed and what has grown at particular grain boundaries at a point in time during the dynamic dissipative process. However, this is not directly relevant to the final mineral assemblage. In an equilibrium context, calculations with equations like (43) give a much more appropriate representation of 
equilibrium coexistence than Wheeler (2014) gives. His suggestion that there is a large effect of non-hydrostatic stress on metamorphic mineral equilibria is erroneous.

As an aside, it is unhelpful of Wheeler (2014) on his second page to repeat that a Gibbs energy cannot be written for non-hydrostatically-stressed solids. As spelt out in the main text in relation to (11), a Gibbs energy defined by $\mathcal{L}_{p \theta} U$ is not available in solid as there is no thermodynamic pressure in solid. But a Gibbs energy can certainly be defined using $G=\mathcal{L}_{\mathbf{T} \theta} U,(11)$. Nor is it correct to assert that a global equilibrium involving non-hydrostatically-stressed solids and fluid is not attainable. The present work, and the papers of Larché and Cahn $(1973,1978,1985)$ on which much of the development is based, show how such an equilibrium should be considered.

\section{Appendix 4: Discussion of Tajčmanová et al. (2014)}

In recent work, Tajčmanová et al. (2014) discuss compositional zoning in a monomineralic plagioclase moat surrounding kyanite in high-grade felsic gneiss. The concentric zoning in the plagioclase ranges from $x=0.22$ at the outer edge of the moat to $x=0.33$ at the inner edge, with $x$ the proportion of anorthite in the plagioclase, as in eq 28. Tajčmanová et al. (2014) propose that the compositional zoning is due to the effect of a pressure gradient across the moat, with chemical equilibrium attained so that chemical potential is constant across the moat. Their approach is discussed here because it is inconsistent with the thermodynamic formulation developed above, (28). Tajčmanová et al. (2014) introduce a chemical potential expression to be considered constant in the proposed equilibrium

$$
\frac{\mu_{\mathrm{ab}}}{m_{\mathrm{ab}}}-\frac{\mu_{\mathrm{an}}}{m_{\mathrm{an}}}
$$

with $m_{\ell}$ being the molecular mass of $\ell$. They then use (44) to calculate the pressure variation across the moat corresponding to the observed $x$ relationships. The postulated equilibrium relation in (44) can be rewritten as

$$
\left(\frac{\mu_{\mathrm{ab}}}{m_{\mathrm{ab}}}-\frac{\mu_{\mathrm{an}}}{m_{\mathrm{an}}}\right)_{A}=\left(\frac{\mu_{\mathrm{ab}}}{m_{\mathrm{ab}}}-\frac{\mu_{\mathrm{an}}}{m_{\mathrm{an}}}\right)_{B}
$$

with $\mathrm{A}$ being in the inner edge of the moat and $\mathrm{B}$ in the outer edge. The development in the present work generates (27), a superficially-similar equilibrium relation to (45), in terms of the $\mu$ of an exchange, $\mu_{\mathrm{ab} a n}$, a consequence of the adoption of the lattice constraint. The significant difference between (27) and (45) is the appearance of the molecular masses in (45). Should the masses be there? 
Whereas mass-based chemical potentials, $\bar{\mu}_{\ell}=\mu_{\ell} / m_{\ell}$, are a notational convenience in writing energetics per unit mass, equilibrium relations are not generally in terms of mass-based chemical potentials. Gibbs, for example, uses mass-based chemical potentials but whenever equilibrium relations are derived, these $\bar{\mu}_{\ell}$ are multiplied by $m_{\ell}$, giving $\mu_{\ell}$, Gibbs (1906), e.g. the interface equilibrium relation, eq. 388.

An equilibrium relation like (45) does arise if an external field that acts on mass, such as a gravitational field, is centred on the kyanite and imposes the pressure variation. External fields are characterised by a potential, $\psi$, a force per unit mass. Augmented chemical potentials, $\mu_{i}+m_{i} \psi$, are then constant in an equilibrium (Guggenheim, 1967, ch. 9; Oppenheim \& Kirkwood, 1961, ch. 15). For a binary plagioclase, separate augmented chemical potentials could then be written for albite and anorthite, involving $\psi$, each of which is constant across the moat. If the two augmented chemical potentials are subtracted, the result is (45). Although Tajčmanová et al. (2015), last paragraph of Sect. 4.1 , alludes to the plagioclase moat in terms of an external field, it is inconceivable that they derive (45) via augmented chemical potentials as there is no external field actually present.

As there is no justification for involving molecular masses in (45), the use of (45) to predict compositional zoning is inappropriate. The observed compositional zoning is far too big to be accounted for by a mean stress gradient caused by variation in non-hydrostatic stress, as shown in the main text. Significant mineral compositional zonings like those around kyanite are most likely due to stranded $\mu$ gradients during mineral assemblage preservation, as argued, for example, by S̆típská et al. (2010).

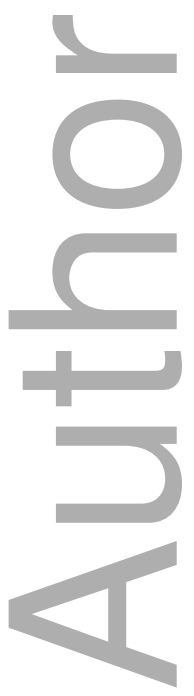




\section{FIGURE CAPTIONS}

Figure 1: Summarising the stress relationships at a solid-fluid grain boundary, using the cross to represent the principal axes of the stress tensor at the indicated point on the boundary, the length of the lines representing the relative magnitude of the principal stresses. The principal stress $t_{3}$, designated to be normal to the grain boundary, equals $-p$ along the boundary, but $t_{1}$ and $t_{2}$ may vary along the boundary.

Figure 2: Schematic depiction of pressure-mean stress relations on grain boundaries involving heterogeneously non-hydrostatically-stressed solids, A, B, C, with fluid, f. (a) location of grain boundaries and fluid grains; (b) thermodynamic pressure $(p)$ and variation of mean stress (as $-\overline{\mathrm{T}}$ ) along the boundaries of $\mathrm{A}$ from $\mathrm{x}$ around to $\mathrm{y}$. The horizontal thick line segments show the fluid grains at a thermodynamic pressure, $\mathrm{p}$. The mean stress around the perimeter of A is the continuous line. The thin line segments show the mean stress in the edge of the adjoining solid grains. The expression for the difference between the mean stress of the solid and the pressure of the fluid is given in the text. For example, at the fluid-A-C grain triple junction, the step of mean stress to $p$ from A to fluid is $\frac{1}{3}\left(q_{1}^{\mathrm{A}}+q_{2}^{\mathrm{A}}\right)$ and from $\mathrm{C}$ to fluid is $\frac{1}{3}\left(q_{1}^{\mathrm{C}}+q_{2}^{\mathrm{C}}\right)$. The mean stress step between $\mathrm{A}$ and $\mathrm{C}$ at the fluid-A-C triple junction is just the difference between these two steps.

Figure 3: Chemical potential relationships in cross-section across several non-hydrostatically-stressed grains in an equilibrium, showing the constancy of the $\mu$ of exchange, $\mu_{(\mathrm{CaAl})_{-1}(\mathrm{NaSi})}$, with pa $=$ paragonite, $\mathrm{ma}=$ margarite, $\mathrm{an}=$ anorthite and $a b=$ albite. The $\mu$ of the additive end-members are only defined in the grain boundaries (dots) and in the fluid (solid line), whereas $\mu_{\mathrm{ex}}$ is defined through all the minerals and fluid and is constant in the equilibrium.

Figure 4: Kyanite-sillimanite surface of equilibrium coexistence, with fluid, in terms of $q_{1}$ and $q_{2}$ for kyanite, at $650^{\circ} \mathrm{C}$. The ellipses are contours of $p$ displacements from the $\mathrm{ky}=$ sill pressure at this temperature with both minerals hydrostatically stressed.

Displacement of $p$, and $q_{1}$ and $q_{2}$ are in kbar. For sillimanite hydrostatically-stressed. For kyanite, $V / \kappa=0.0028 \mathrm{~kJ} \mathrm{kbar}^{-2}$ and $V / \mu=0.0042 \mathrm{~kJ} \mathrm{kbar}^{-2}$. The $p$ displacements are very small compared to $2 \sigma_{p}=0.26$ kbar with dataset uncertainties, calculated for both minerals hydrostatically-stressed.

Figure 5: Kyanite-sillimanite surface of equilibrium coexistence, with fluid, at $650^{\circ} \mathrm{C}$ with both kyanite and sillimanite non-hydrostatically stressed, using (25) and simplifying 
with $q_{1}^{\mathrm{ky}}=q_{2}^{\mathrm{ky}}=q^{\mathrm{ky}}$ and $q_{1}^{\text {sill }}=q_{2}^{\text {sill }}=q^{\text {sill }}$. The hyperbolae are for $p$ displacements in $\mathrm{kbar}$ from the $\mathrm{ky}=$ sill pressure at this temperature if both minerals are hydrostatically stressed. For kyanite, $V / \kappa=0.0019 \mathrm{~kJ} \mathrm{kbar}^{-2}$ and $V / \mu=0.0042 \mathrm{~kJ} \mathrm{kbar}^{-2}$ and for sillimanite, $V / \kappa=0.0030 \mathrm{~kJ} \mathrm{kbar}^{-2}$ and $V / \mu=0.0054 \mathrm{~kJ} \mathrm{kbar}^{-2}$.

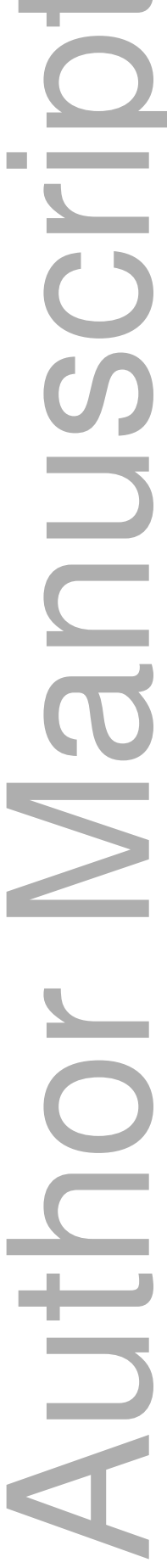


Figure 1:

Figure 2:

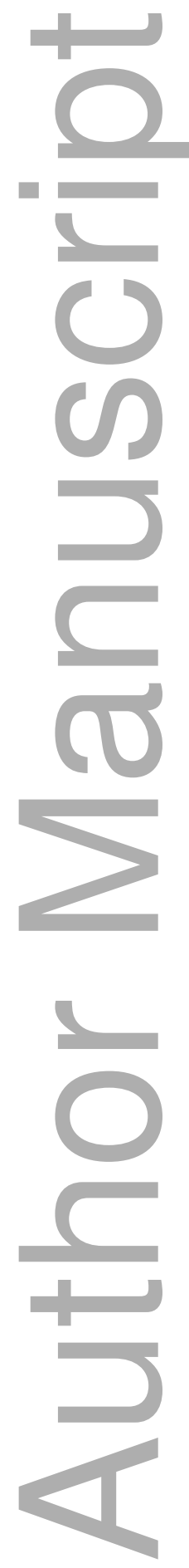

Figure 3:

This article is protected by copyright. All rights reserved 


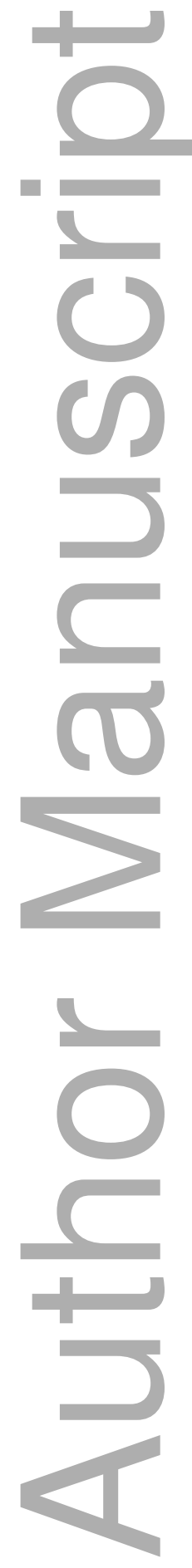

Figure 4:

Figure 5:

This article is protected by copyright. All rights reserved 
$t_{3}=-p \quad$ jmg_12298_f1.pdf

1

$t_{1}, t_{2}$ unconstrained

$\mathrm{t}_{2}$ out of page

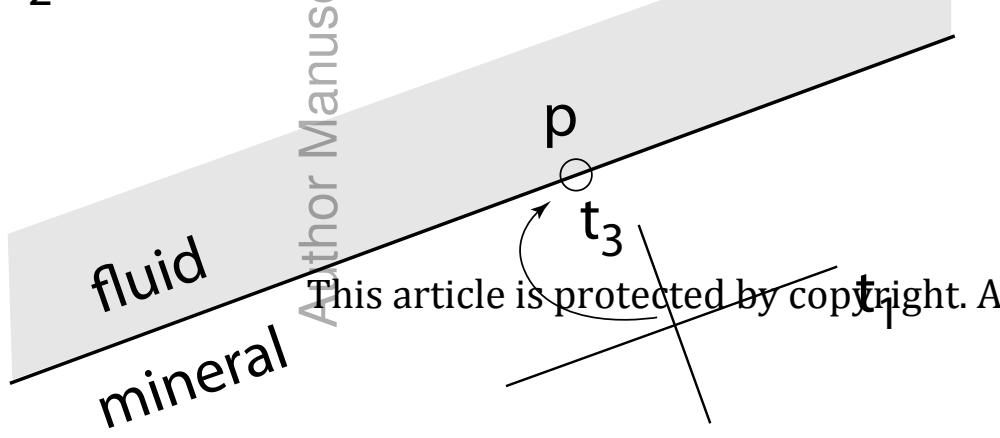


jmg_12298_f3.pdf

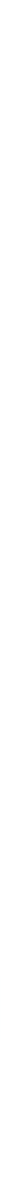




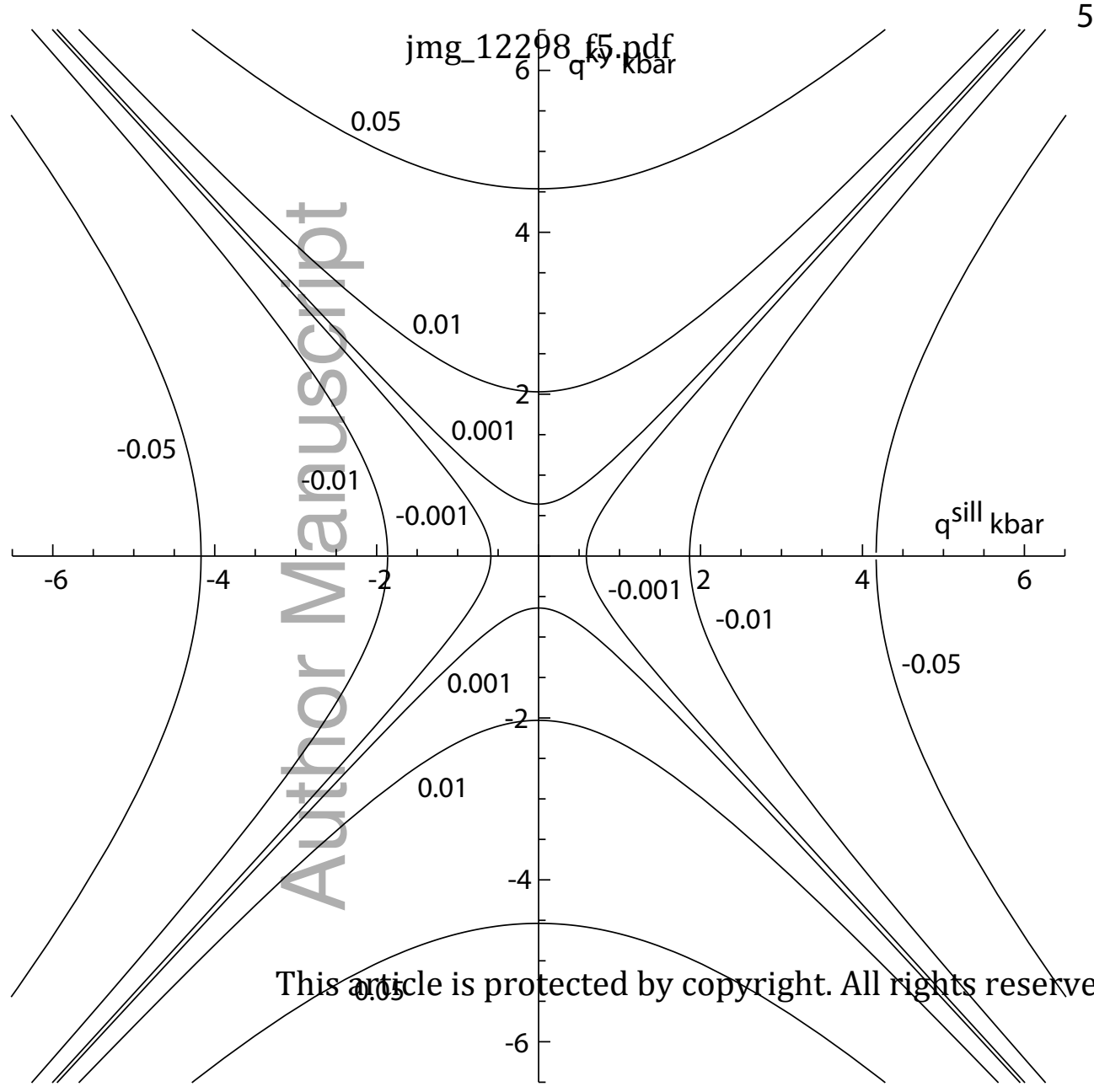




\section{University Library}

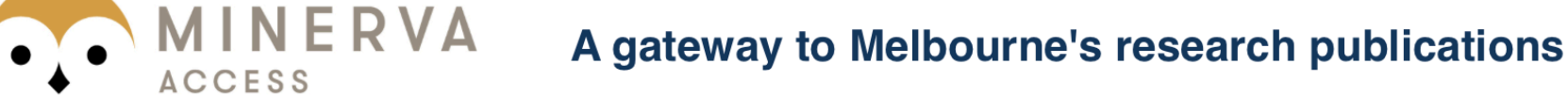

Minerva Access is the Institutional Repository of The University of Melbourne

Author/s:

Powell, R;Evans, KA;Green, ECR;White, RW

Title:

On equilibrium in non-hydrostatic metamorphic systems

Date:

2018-05-01

Citation:

Powell, R., Evans, K. A., Green, E. C. R. \& White, R. W. (2018). On equilibrium in nonhydrostatic metamorphic systems. JOURNAL OF METAMORPHIC GEOLOGY, 36 (4), pp.419-438. https://doi.org/10.1111/jmg.12298.

Persistent Link:

http://hdl.handle.net/11343/283804 